\title{
On the topology of fillings of contact manifolds and applications
}

\author{
Alexandru Oancea and Claude Viterbo*
}

\begin{abstract}
The aim of this paper is to address the following question: given a contact manifold $(\Sigma, \xi)$, what can be said about the symplectically aspherical manifolds $(W, \omega)$ bounded by $(\Sigma, \xi)$ ? We first extend a theorem of Eliashberg, Floer and McDuff to prove that, under suitable assumptions, the map from $H_{*}(\Sigma)$ to $H_{*}(W)$ induced by inclusion is surjective. We apply this method in the case of contact manifolds admitting a contact embedding in $\mathbb{R}^{2 n}$ or in a subcritical Stein manifold. We prove in many cases that the homology of the fillings is uniquely determined. Finally, we use more recent methods of symplectic topology to prove that, if a contact hypersurface has a subcritical Stein filling, then all its SAWC fillings have the same homology.

A number of applications are given, from obstructions to the existence of Lagrangian or contact embeddings, to the exotic nature of some contact structures. We refer to the table in Section 7 for a summary of our results.
\end{abstract}

Mathematics Subject Classification (2010). 53D35, 57R40, 32S20.

Keywords. Topology of symplectic fillings of contact manifolds, obstructions to contact embeddings.

\section{Introduction}

In this paper all symplectic manifolds will be assumed to be connected, of dimension $2 n$, and symplectically aspherical, meaning that the symplectic form vanishes on the second homotopy group. All contact manifolds are connected and have dimension $2 n-1$. We denote by $\sigma_{0}$ the standard symplectic form on $\mathbb{R}^{2 n}$ or $\mathbb{C} P^{n}$, and by $\alpha_{0}$ the standard contact form on the sphere $S^{2 n-1}$.

In a celebrated paper ([McD]), Eliashberg, Floer and McDuff proved that, if $(W, \omega)$ is a symplectically aspherical manifold with contact boundary $\left(S^{2 n-1}, \alpha_{0}\right)$, then $W$ is diffeomorphic to the unit ball $B^{2 n}$. In the case of dimension 4 , Gromov had earlier proved ([Gr]) that $W$ is actually symplectomorphic to $\left(B^{4}, \sigma_{0}\right)$, but this

\footnotetext{
* Supported by ANR project "Floer Power" ANR-08-BLAN-0291-03/04. We are grateful to Beijing International Center for Mathematical Research of Beijing University for hospitality during the completion of this paper.
} 
relies heavily on positivity of intersection for holomorphic curves that is special to dimension 4.

One can ask more generally, given a fillable contact manifold $(\Sigma, \xi)$ and a symplectically aspherical filling $(W, \omega)$, what can be said about the topology or the homology of $W$. Is it uniquely determined by the contact structure $(\Sigma, \xi)$ ? Is it determined by the topology of $\Sigma$ ? Do we have lower bounds? Upper bounds? It turns out that all these possibilities actually occur.

For example, if $(\Sigma, \xi)$ has a contact embedding into $\left(\mathbb{R}^{2 n}, \sigma_{0}\right)$ - many such examples can be found in [La] - it readily follows from the Eliashberg-Floer-McDuff theorem and some elementary algebraic topology that all subcritical Stein fillings have the same homology. If the homology of $\Sigma$ vanishes in degree $n$, we can prove that all Stein fillings have the same homology. This gives easy examples of contact manifolds with no contact embedding in $\left(\mathbb{R}^{2 n}, \sigma_{0}\right)$. As far as the authors know, there are only few previously known examples of fillable manifolds not embeddable in $\mathbb{R}^{2 n}$, with the exception of recent results in [C-F-O] and [A-McL], which however assume the exactness of the embeddings, an assumption we usually can dispense with. More general results of the same homological flavour follow from the same methods, and a generalization of the Eliashberg-Floer-McDuff theorem to the case of subcritical Stein manifolds. These are manifolds $W$ admitting an exhausting plurisubharmonic function with no critical points of index $n=\frac{1}{2} \operatorname{dim}(W)$ (see Definition 2.4).

Our last result uses more sophisticated tools. One of them will be symplectic homology of $W$, and its positive part, defined in [V]. It turns out that this positive part, under mild assumptions on the Conley-Zehnder index of closed characteristics, is independent of the filling. This is proved in [C-F-O] as a consequence of arguments in [B-O-1]. A symplectically aspherical manifold $(W, \omega)$ with contact type boundary is called an SAWC-manifold if its symplectic homology vanishes (this is equivalent to the Strong Algebraic Weinstein Conjecture formulated in [V], cf. Section 5). We show that, if $(\Sigma, \xi)$ bounds a subcritical Stein manifold $(W, \omega)$, any other SAWC filling will have the same homology as $W$.

Of course many questions remain open. As far as we can see, nothing can be said about the symplectic topology of fillings outside the subcriticality/non-subcriticality alternative. Are there examples of compact manifolds $L$ such that $S T^{*} L$ has fillings with homology different from $H_{*}(L)$ ? Is there an embedding of the Brieskorn sphere of a singularity of Milnor number $\mu$ in the Milnor fibre of a singularity of Milnor number $\mu^{\prime}<\mu$ ?

\section{The Eliashberg-Floer-McDuff theorem revisited}

Conventions. We denote by $(W, \omega)$ a symplectic manifold of dimension $2 n$ which is symplectically aspherical $\left([\omega] \pi_{2}(W)=0\right)$. We denote by $(\Sigma, \xi)$ a contact manifold of dimension $2 n-1$. We assume that $\xi$ is co-orientable, and fix a co-orientation. The contact structure $\xi$ is then defined by a contact form $\alpha$, and $\Sigma$ is oriented by 
$\alpha \wedge(d \alpha)^{n-1} \neq 0$. All homology and cohomology groups are taken with coefficients in a field.

Definition 2.1. A contact embedding of $(\Sigma, \xi)$ in $(W, \omega)$ is a codimension 1 embedding such that there exists a positive contact form $\alpha$ extending to a neighbourhood of $\Sigma$ as a primitive of $\omega$. The contact embedding is called exact if $\alpha$ extends to the whole of $W$ as a primitive of $\omega$.

Definition 2.2. A (co-oriented) hypersurface $\Sigma \subset(W, \omega)$ is said to be of contact type in $W$ if there exists a primitive $\alpha$ of $\omega$, defined in a neighbourhood of $\Sigma$, and restricting on $\Sigma$ to a contact form (whose $\omega$-dual vector field defines the positive co-orientation of $\Sigma$ ). The hypersurface is said to be of restricted contact type in $W$ if there exists such a primitive $\alpha$ which is globally defined on $W$.

Definition 2.3. A symplectic filling of $(\Sigma, \xi)$ is a symplectic manifold $(W, \omega)$ without closed components, such that $\partial W=\Sigma$ and there exists a positive contact form $\alpha$ extending to a neighbourhood of $\Sigma$ as a primitive of $\omega$. We shall say that the symplectic filling is exact if $\alpha$ extends to the whole of $W$ as a primitive of $\omega$.

Definition 2.4. A symplectic filling $(W, \omega)$ of $(\Sigma, \xi)$ is a Stein filling if $W$ has a complex structure $J$, and a non-positive plurisubharmonic function $\psi$, such that $\Sigma=\psi^{-1}(0)$ and $-J^{*} d \psi$ is a contact form defining $\xi$. Note that $\psi$ can always be chosen to be a Morse function. Then its critical points have index at most $n$, so that $W$ has the homotopy type of a CW complex of dimension $\leq n$. If we can find the function $\psi$ with no critical points of index $n$, then $W$ is said to be subcritical Stein.

Remark 2.5. A contact embedding of $(\Sigma, \xi)$ in $(W, \omega)$ which is separating - i.e. $W \backslash \Sigma$ consists of two connected components - yields a filling of $(\Sigma, \xi)$ by the connected component of $W \backslash \Sigma$ for which the boundary orientation of $\Sigma$ coincides with the orientation induced by a positive contact form $\alpha$. This filling we shall call the interior of $\Sigma$ and we shall denote by $Z$. If $W$ is non-compact, $Z$ is the bounded component of $W \backslash \Sigma$. Note that $\Sigma$ is always separating if $H_{2 n-1}(W ; \mathbb{Z})$ is torsion, and in particular if $W$ is Stein and $n \geq 2$.

Our goal in this section is to prove the following theorem.

Theorem 2.6. Assume $(\Sigma, \xi)$ admits a contact embedding in a subcritical Stein manifold $\left(M, \omega_{0}\right)$, with interior component $Z$. Let $(W, \omega)$ be a symplectically aspherical filling of $\Sigma$ and assume that one of the following two conditions is satisfied:

(a) $H_{2}(W, \Sigma)=0$;

(b) $\Sigma$ is simply connected. 
Then the map

$$
H_{j}(\Sigma) \longrightarrow H_{j}(W)
$$

induced by inclusion is onto in every degree $j \geq 0$.

Remark 2.7. Condition (a) holds if $W$ is Stein of dimension $2 n \geq 6$. The embedding $\Sigma \hookrightarrow M$ is always separating since $H_{2 n-1}(M ; \mathbb{Z})=0$.

Remark 2.8. When $\Sigma$ is a sphere, we get that $W$ has vanishing homology. This is the original Eliashberg-Floer-McDuff theorem (see [McD]), since an application of the $h$-cobordism theorem, plus the fact - due to Eliashberg - that $\pi_{1}(W)$ vanishes, implies that $W$ is diffeomorphic to the ball $B^{2 n}$. Indeed, since $H_{j}\left(S^{2 n-1}\right)=0$ for $1 \leq j \leq 2 n-2$, the same holds for $H_{j}(W)$. In particular $H_{1}(W)=0$, which implies that $H^{1}(W)$ and $H_{2 n-1}(W)$ vanish. When $n=2$ Gromov (see [Gr]) proved that $W$ is symplectomorphic to the ball $B^{4}$, but this relies heavily on purely 4-dimensional arguments (positivity of intersection of holomorphic curves).

Our proof of Theorem 2.6 closely follows the original proof in [McD], except for the final homological argument.

We shall start by working in the following special situation, and we will then prove that this is enough to deal with the general case.

Let $\left(P, \omega_{P}\right)$ be a symplectic manifold and $H$ be a codimension two symplectic submanifold of $P$.

We consider the symplectic manifold $\left(P \times S^{2}, \omega_{P} \oplus \sigma\right)$, where $\sigma$ is the standard symplectic form on $S^{2}$ normalized by $[\sigma]\left[S^{2}\right]=1$. Viewing $S^{2}$ as $\mathbb{C} \cup\{\infty\}$ we denote $D_{-}^{2}:=\left\{z:|z|<\frac{1}{2}\right\}$ and $D_{+}^{2}:=\left\{z:|z|>\frac{1}{2}\right\}$. Let $(\Sigma, \xi)$ be a separating contact manifold contained in $(P \backslash H) \times D_{-}^{2}$, with interior $Z$. We set $Y=\left(P \times S^{2} \backslash Z\right)$ and

$$
V=Y \sqcup_{\Sigma} W=\left(P \times S^{2} \backslash Z\right) \sqcup_{\Sigma} W,
$$

where $(W, \omega)$ is a filling of $(\Sigma, \xi)$ (Figure 1). We assume that the contact 1 -forms on $\Sigma$ viewed as a contact hypersurface in $P \times S^{2}$ and $W$ agree, and denote them by $\alpha$. Then $V$ has a symplectic form $\omega_{V}$ obtained by gluing $\omega_{P} \oplus \sigma$ on $Y$ and $\omega$ on $W$.

Let $p_{0}$ be a point in $H$ and denote $A:=\left[\left\{p_{0}\right\} \times S^{2}\right] \in H_{2}(V ; \mathbb{Z})$ (note that, for $p_{0} \in H$, we have that $\left.\left\{p_{0}\right\} \times S^{2} \subset Y \subset V\right)$. Given an $\omega_{V}$-compatible almost complex structure $J$ on $V$, we denote by $\widetilde{\mathcal{M}}_{J}$ the space of $J$-holomorphic maps $u: \mathbb{C} P^{1} \rightarrow V$ representing the class $A$.

Lemma 2.9. Assume the symplectic form $\omega_{P}$ is integral, i.e. $\left[\omega_{P}\right] \in \operatorname{Im}\left(H^{2}(P ; \mathbb{Z}) \rightarrow\right.$ $\left.H^{2}(P ; \mathbb{R})\right)$. Assume also that one of the following conditions holds:

(a) $H_{2}(W, \Sigma)=0$;

(b) $\Sigma$ is simply connected. 
Then, for any $\omega_{V}$-compatible almost complex structure $J$ on $V$, the class $A$ is $J$ simple, meaning that it cannot be decomposed as $A=B+C$, with $B, C \in \mathrm{H}_{2}(V ; \mathbb{Z})$ represented by non-constant $J$-holomorphic spheres.

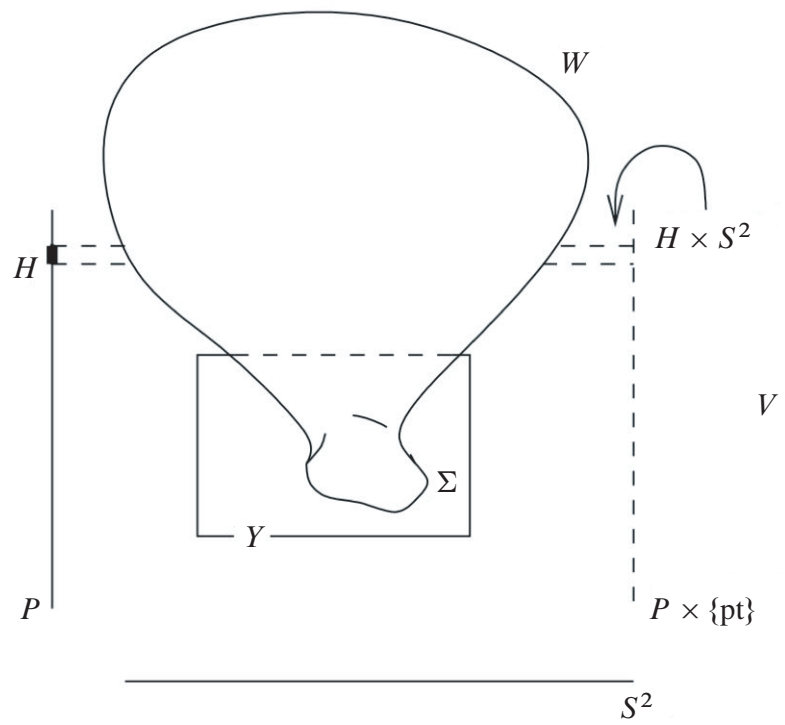

Figure 1. Geometric setup.

Proof. Arguing by contradiction, let us assume that such a decomposition exists for some $\omega_{V}$-compatible $J$. We obtain in particular $0<\omega_{V}(B), \omega_{V}(C)<\omega_{V}(A)$. Let $\Gamma_{B}$ and $\Gamma_{C}$ be the $J$-holomorphic spheres representing $B$ and $C$. Since $W$ is symplectically aspherical, it is not possible that any of the cycles $\Gamma_{B}, \Gamma_{C}$ be entirely contained in $W$. Since $\omega_{P}$ is integral and $\int_{S^{2}} \sigma=1$, it follows that the class $A$ has minimal area in $P \times S^{2}$, so that it is neither possible that any of the cycles $\Gamma_{B}, \Gamma_{C}$ be entirely contained in $Y \subset P \times S^{2}$. Thus $\Gamma_{B}$ and $\Gamma_{C}$ intersect both $Y$ and $W$. By (smoothly) perturbing the representing $J$-holomorphic spheres, we can achieve transverse intersection with $\Sigma$, along some collection of circles.

Let us now assume (a). We consider the two pieces in $\Gamma_{C}$ separated by $\Sigma$. We denote by $C_{1}$ the piece in $W$ and by $C_{2}$ its complement. Then $\int_{\Gamma_{C}} \omega_{V}=$ $\int_{C_{1}} \omega_{V}+\int_{C_{2}} \omega_{V}$. But $C_{1} \in H_{2}(W, \Sigma)$ so by our assumption there is a cycle $\Gamma$ in $\Sigma$ such that $\partial C_{1}=\partial \Gamma$. Because $C_{1} \cup \Gamma$ is a cycle in $H_{2}(W)$, and the map $H_{2}(\Sigma) \rightarrow H_{2}(W)$ is onto (using again $H_{2}(W, \Sigma)=0$ ), we obtain that $C_{1} \cup \Gamma$ is homologous in $W$ to a cycle $C_{3}$ contained in $\Sigma$. Thus $\int_{C_{1}} \omega_{V}-\int_{\Gamma} \omega_{V}=\int_{C_{3}} \omega_{V}$, which vanishes because $\omega_{V}$ is exact near $\Sigma$. Finally $C_{2} \cup \Gamma$ is a cycle in $Y$ with the 
same area as $\Gamma_{C}=C_{1} \cup C_{2}$. Since $Y \subset P \times S^{2}$, this contradicts the fact that the class $A$ has minimal area in $P \times S^{2}$.

We now assume (b). As above, let $C_{1}, C_{2}$ be the parts of $\Gamma_{C}$ separated by $\Sigma$, with $C_{1}$ being the piece contained in $W$. By assumption, we can cap all the common boundary circles of $C_{1}$ and $C_{2}$ by discs. Let us denote this union of discs by $\Gamma$. Then $C_{1} \cup \Gamma$ is a collection of spheres in $W$ and, by symplectic asphericity, it has zero area. Thus $C_{2} \cup \Gamma$ is a cycle in $Y$ with the same area as $\Gamma_{C}=C_{1} \cup C_{2}$. This contradicts again the minimality of the area of the class $A$ in $P \times S^{2}$.

As a consequence of Lemma 2.9 we have the following facts:

- For any $\omega_{V}$-compatible almost complex structure $J$ on $V$, the elements of $\tilde{\mathcal{M}}_{J}$ are simple curves (i.e. they are not multiply covered).

- For a generic choice of the $\omega_{V}$-compatible almost complex structure $J$, the linearized Cauchy-Riemann operator is surjective for every element of $\widetilde{\mathcal{M}}_{J}$ and $\widetilde{\mathcal{M}}_{J} / \operatorname{PSL}(2, \mathbb{C})$ is a smooth manifold of dimension

$$
\operatorname{dim} \tilde{\mathcal{M}}_{J} / \operatorname{PSL}(2, \mathbb{C})=2 n+2\left\langle c_{1}(V), A\right\rangle-6=2 n-2 .
$$

Such an almost complex structure $J$ is called regular.

- The manifold $\tilde{\mathcal{M}}_{J} / \operatorname{PSL}(2, \mathbb{C})$ is compact.

Let $J$ be a regular almost complex structure for which $P \times\{z\}, z=-1,1, \infty$ are $J$-complex submanifolds. It is convenient to consider the following model for the manifold $\widetilde{\mathcal{M}}_{J} / \operatorname{PSL}(2, \mathbb{C})$. Given (disjoint) submanifolds $T_{-1}, T_{1}, T_{\infty} \subset V$ that are $C^{2}$-close to $P \times\{-1\}_{2} P \times\{1\}$, respectively $P \times\{\infty\}$, we denote by $\mathcal{M}:=\mathcal{M}_{J}$ the set of elements $u \in \tilde{\mathcal{M}}_{J}$ such that $u(z) \in T_{z}, z=-1,1, \infty$. For a generic choice of the perturbations $T_{z}, z=-1,1, \infty$ the relevant evaluation maps are transverse, so that $\mathcal{M}$ is a compact submanifold of $\widetilde{\mathcal{M}}_{J}$ of dimension $2 n-2$. By positivity of intersections (see also Step 1 in the proof of Lemma 2.10 below), we infer that $\mathcal{M}$ intersects every orbit of $\operatorname{PSL}(2, \mathbb{C})$ exactly once, and it follows that the natural map $\mathcal{M} \rightarrow \widetilde{\mathcal{M}}_{J} / \operatorname{PSL}(2, \mathbb{C})$ is a diffeomorphism.

Given an $\omega_{P}$-compatible almost complex structure $J_{P}$ on $P$, we denote by $\tilde{J}_{P}$ the almost complex structure $J_{P} \oplus i$ on $P \times S^{2}$. For the proof of the next result, we closely follow [McD], pp. 660-661.

Let $J_{P}$ be an $\omega_{P}$-compatible almost complex structure on $P$. We say that $H$ is hyperplane section-like for $J_{P}$ if the following hold:

- $H$ is a $J_{P}$-complex submanifold,

- there exists a codimension two $J_{P}$-complex (singular) submanifold $B \subset H$ (the base locus), a relatively compact neighbourhood $U$ of $H$ and a relatively compact open neighbourhood $U_{B}$ of $B$, and a family $\mathscr{H}_{z}$ of $J_{P}$-complex hypersurfaces parametrized by an open neighbourhood of 0 in $\mathbb{C}$ and contained in $\mathcal{U}$, such that $\mathscr{H}_{z} \cap\left(\mathcal{U} \backslash \bar{U}_{B}\right)$ foliate $U \backslash \bar{U}_{B}$. 
Note that the hyperplane section of a projective manifold is hyperplane sectionlike for the underlying complex structure.

Lemma 2.10. Let $J_{P}$ be an $\omega_{P}$-compatible almost complex structure on $P$ such that $H$ is hyperplane section-like for $J_{P}$. For every regular $J$ which is close to $\tilde{J}_{P}$ on a neighbourhood of $H \times S^{2} \subset V$, the evaluation map

$$
\mathrm{ev}: \mathcal{M} \times S^{2} \rightarrow V
$$

has degree \pm 1 .

Proof. Let $B$ be the base locus for $H$ and let $U, \mathcal{U}_{B}$ be the neighbourhoods of $H$ and $B$ such that $U \backslash \bar{U}_{B}$ is foliated by $J_{P}$-complex hypersurfaces $\mathscr{H} \cap\left(\mathcal{U} \backslash \bar{U}_{B}\right)$. We consider $U \times S^{2}$ as a neighbourhood of $H \times S^{2}$ in $V$, so that $\left(\mathcal{U} \backslash \bar{U}_{B}\right) \times S^{2}$ is also foliated by the $\tilde{J}_{P}$-complex hypersurfaces $\left(\mathcal{H} \times S^{2}\right) \cap\left(\mathcal{U} \backslash \bar{U}_{B}\right) \times S^{2}$. We prove the lemma in three steps.

Step 1: Let $J$ coincide with $\tilde{J}_{P}$ on $U \times S^{2}$. For every $p \in\left(\mathcal{U} \backslash \bar{U}_{B}\right) \times S^{2}$, there exists a unique element of $\widetilde{\mathcal{M}}_{J} / \operatorname{PSL}(2, \mathbb{C})$ through $p$.

Let $\mathscr{H}_{p} \times S^{2}$ be the $\tilde{J}_{P}$-complex hypersurface through $p$. Given a curve $C$ through $p$ represented by some $[u] \in \widetilde{\mathcal{M}}_{J} / \operatorname{PSL}(2, \mathbb{C})$, the homological intersection between $C$ and $\mathscr{H}_{p} \times S^{2}$ is zero. Since $C \cap \mathscr{H}_{p} \times S^{2} \neq \emptyset$, it follows from the positivity of intersections for holomorphic curves that $C$ is entirely contained in $\mathscr{H}_{p} \times S^{2}$. But, in $\mathscr{H}_{p} \times S^{2}$, there is clearly a unique $\tilde{J}_{P}$-holomorphic curve in the class $\{\mathrm{pt}\} \times S^{2}$ through each point. (Remark: Positivity of intersections is proved with all details in a 4-dimensional context in [L-McD-S]. The higher dimensional case of a curve intersecting a complex hypersurface is treated using exactly the same methods.)

Step 2: Let $J_{0}$ be an almost complex structure which coincides with $\tilde{J}_{P}$ on $U \times S^{2}$. For every $J$ which is close enough to $J_{0}$, and for every point $p \in\left(\mathcal{U} \backslash \bar{U}_{B}\right) \times S^{2}$, there is a unique element of $\widetilde{\mathcal{M}}_{J} / \operatorname{PSL}(2, \mathbb{C})$ through $p$.

We follow [McD], Lemma 3.5. Arguing by contradiction, we find a point $p \in$ $\left(u \backslash \bar{U}_{B}\right) \times S^{2}$ and a sequence $J_{v}, v \geq 1$ converging to $J_{0}$ such that, for every $v$, there exist two distinct unparametrized $J_{v}$-holomorphic spheres $C_{v}$ and $C_{v}^{\prime}$ through $p$. Since $A$ is a simple class (Lemma 2.9), both $C_{v}$ and $C_{v}^{\prime}$ converge as unparametrized spheres to the unique $J_{0}$-holomorphic sphere $C$ through $p$. In particular, for $v$ large enough they are both contained in $U^{\prime} \times S^{2}$, for some relatively compact open subset $u^{\prime} \subset u$.

We now view $U \times S^{2}$ as a subset of $P \times S^{2}$ and extend $\left.J_{v}\right|_{u^{\prime} \times S^{2}}$ to an almost complex structure $J_{v}^{\prime}$ on $P \times S^{2}$ which is compatible with $\omega_{P} \oplus \sigma$ and satisfies $J_{v}^{\prime} \rightarrow \tilde{J}_{P}, v \rightarrow \infty$. For $v$ large enough, the $J_{v}$-holomorphic curves $C_{v}, C_{v}^{\prime}$ passing through $p$ are now viewed in $P \times S^{2}$, where they are $J_{v}^{\prime}$-holomorphic. The almost 
complex structure $\tilde{J}_{P}$ is obviously regular for curves in the class $\left[\{\mathrm{pt}\} \times S^{2}\right]$ in $P \times S^{2}$, and Step 1 shows that the evaluation map

$$
\mathrm{ev}: \tilde{\mathcal{M}}_{\tilde{J}_{P}} \times_{\mathrm{PSL}(2, \mathbb{C})} S^{2} \longrightarrow P \times S^{2}
$$

is a diffeomorphism (we make a slight abuse of notation and write here $\tilde{\mathcal{M}}_{J}$ for the space of $J$-holomorphic curves in $P \times S^{2}$ representing the class $\left.\left[\{\mathrm{pt}\} \times S^{2}\right]\right)$. The evaluation map remains a diffeomorphism for small perturbations of $\tilde{J}_{P}$, and we reach a contradiction with the fact that $p$ has at least two preimages via the evaluation maps ev: $\widetilde{\mathcal{M}}_{J_{v}^{\prime}} \times_{\mathrm{PSL}(2, \mathbb{C})} S^{2} \rightarrow P \times S^{2}$ for $v$ large enough.

Step 3: We prove the lemma.

The degree of the evaluation map can be computed by counting the number of preimages of a generic point in $V$. We can therefore choose our point generically in $\left(U \backslash \vec{U}_{B}\right) \times S^{2} \subset V$, and the number of preimages is then equal to one by Step 2 .

Proposition 2.11. Let $\left(P, \omega_{P}\right)$ be a symplectic manifold such that $\omega_{P}$ is an integral class, and let $H \subset P$ be a codimension two symplectic submanifold which is hyperplane section-like for some $\omega_{P}$-compatible almost complex structure $J_{P}$. Let $(\Sigma, \xi)$ be a contact separating hypersurface of $\left(P \times S^{2}, \omega_{P} \oplus \sigma\right)$ which is contained in $(P \backslash H) \times S^{2}$. Let $W$ be any symplectically aspherical filling of $(\Sigma, \xi)$. Assume one of the following two conditions holds:

(a) $H_{2}(W, \Sigma)=0$;

(b) $\Sigma$ is simply connected.

Then the map

$$
H_{j}(\Sigma) \longrightarrow H_{j}(W)
$$

induced by inclusion is surjective.

Proof. We can assume without loss of generality that the contact forms induced on $\Sigma$ viewed as contact hypersurface in $W$ and respectively in $P \times S^{2}$ are the same. Indeed, if $\alpha$ denotes the contact form coming from the contact embedding in $W$, and $\beta$ denotes the contact form coming from the contact embedding in $P \times S^{2}$, we can modify $W$ by attaching a large piece of symplectization $([1, R] \times \Sigma, d(r \alpha)), R \gg 1$ inside which we can find a graph over $\Sigma$ on which the induced contact form is a large multiple of $\beta$. By removing what lies beyond the graph and rescaling the symplectic form, we reduce ourselves to the situation where $\alpha=\beta$.

We are now in a position to perform the construction of $V$ described above: we take away the interior $Z$ of $\Sigma$ in $P \times S^{2}$, and we replace it with $W$. Lemmas 2.9 and 2.10 hold true, and there exists an $\omega_{V}$-compatible regular almost complex structure $J$ on $V$ which satisfies the assumptions of Lemma 2.10. The outcome is the compact manifold $\mathcal{M}=\mathcal{M}_{J}$ together with the degree \pm 1 map ev: $\mathcal{M} \times S^{2} \rightarrow V$. 
Given the sets $S \subset T$, let us denote by $i_{T}^{S}$ the inclusion map of $S$ into $T$. Let $C_{1} \in H_{j}(W)$ be fixed. In order to prove that $C_{1}$ lies in the image of $\left(i_{W}^{\Sigma}\right)_{*}$, it is enough to show that $C:=\left(i_{V}^{W}\right)_{*}\left(C_{1}\right) \in H_{j}(V)$ lies in the image of $\left(i_{V}^{Y}\right)_{*}$. Indeed, the Mayer-Vietoris exact sequence is

$\longrightarrow H_{j}(\Sigma) \stackrel{\left(i_{W}^{\Sigma}\right)_{*} \oplus\left(i_{Y}^{\Sigma}\right)_{*}}{\longrightarrow} H_{j}(W) \oplus H_{j}(Y) \stackrel{\left(i_{V}^{W}\right)_{*}-\left(i_{V}^{Y}\right)_{*}}{\longrightarrow} H_{j}(V) \longrightarrow H_{j-1}(\Sigma) \longrightarrow$.

Thus, if there is $C_{2} \in H_{j}(Y)$ such that $C=\left(i_{V}^{Y}\right)_{*}\left(C_{2}\right)$, then $\left(C_{1}, C_{2}\right)$ is in the kernel of $\left(i_{V}^{W}\right)_{*}-\left(i_{V}^{Y}\right)_{*}$, hence in the image of $\left(i_{W}^{\Sigma}\right)_{*} \oplus\left(i_{Y}^{\Sigma}\right)_{*}$. In particular $C_{1}$ is in the image of $\left(i_{W}^{\Sigma}\right)_{*}$.

We now prove that $C$ lies in the image of $\left(i_{V}^{Y}\right)_{*}$. We start with the observation that the map

$$
(\mathrm{ev})_{*}: H_{j}\left(\mathcal{M} \times S^{2}\right) \longrightarrow H_{j}(V)
$$

is surjective. Since we use field coefficients, this is equivalent to injectivity of ev* in cohomology, which in turn follows from the non-degeneracy of the cup-product pairing and the fact that ev: $\mathcal{M} \times S^{2} \rightarrow V$ has non-zero degree (with respect to any field of coefficients). We can therefore write $C=\operatorname{ev} *\left(\Gamma_{C}\right)$ for some $\Gamma_{C} \in$ $H_{j}\left(\mathcal{M} \times S^{2}\right)$ or, equivalently,

$$
C=\mathrm{ev}_{*}\left(A \otimes\{\mathrm{pt}\}+B \otimes\left[S^{2}\right]\right)
$$

for some $A \in H_{j}(\mathcal{M})$ and $B \in H_{j-2}(\mathcal{M})$.

We claim that $B$ must vanish. Arguing by contradiction and using that $\mathcal{M}$ is orientable, let $B^{\prime}$ be Poincaré dual to $B$ in $H_{*}(\mathcal{M})$, so that $B \cdot B^{\prime}=\{\mathrm{pt}\}$. We obtain

$$
\Gamma_{C} \cdot\left(B^{\prime} \otimes\{\mathrm{pt}\}\right)=\left(B \otimes\left[S^{2}\right]\right) \cdot\left(B^{\prime} \otimes\{\mathrm{pt}\}\right)=\left(B \cdot B^{\prime}\right) \otimes\{\mathrm{pt}\}=\{\mathrm{pt}\} \otimes\{\mathrm{pt}\} .
$$

This implies that

$$
\{\mathrm{pt}\}=(\mathrm{ev})_{*}\left(\Gamma_{C} \cdot\left(B^{\prime} \otimes\{\mathrm{pt}\}\right)\right)=C \cdot(\mathrm{ev})_{*}\left(B^{\prime} \otimes\{\mathrm{pt}\}\right)=C \cdot \mathrm{ev}_{*}^{\infty}\left(B^{\prime}\right),
$$

where $\operatorname{ev}^{z}(u)=u(z)$. Since $\operatorname{ev}_{*}^{\infty}\left(B^{\prime}\right) \subset P \times\{\infty\}$, we get $C \cdot \mathrm{ev}_{*}^{\infty}\left(B^{\prime}\right)=0$, a contradiction.

As a result, we obtain $C=\operatorname{ev}_{*}^{z}(A)$ (for any $z \in S^{2}$ ), with $A \in H_{j}(\mathcal{M})$. Choosing $z=\infty$ we get that $C \in\left(i_{V}^{P \times\{\infty\}}\right)_{*}\left(H_{j}(P)\right) \subset\left(i_{V}^{Y}\right)_{*}\left(H_{j}(Y)\right)$. This concludes the proof.

Remark 2.12. If the image of the boundary map $H_{j+1}(Y, \Sigma) \rightarrow H_{j}(\Sigma)$ coincides with the image of the boundary map $H_{j+1}(W, \Sigma) \rightarrow H_{j}(\Sigma)$, then $\operatorname{dim} H_{j}(W) \leq$ $\operatorname{dim} H_{j}(P)$. Indeed, it follows from the commutative diagram below that the map $H_{j}(W) \rightarrow H_{j}(V)$ is injective. Since its image is contained in $\operatorname{Im}\left(i_{V}^{P \times\{\infty\}}\right)_{*}$, the 
conclusion follows.

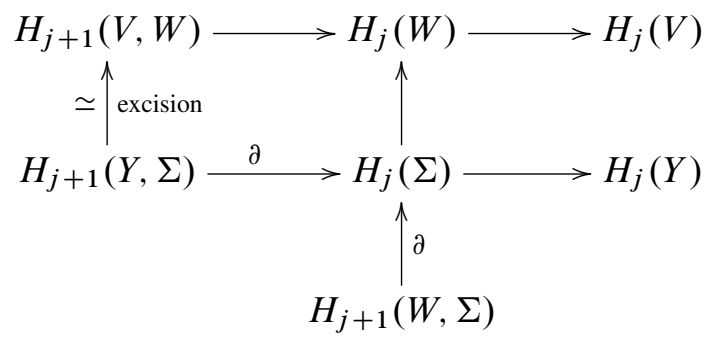

Proof of Theorem 2.6. We use a result of Cieliebak (see [C1]) stating that a subcritical Stein manifold is symplectomorphic to $N \times \mathbb{C}$ where $N$ is Stein, and a result of Lisca and Matić ([Li-M], Section 3, Theorem 3.2), stating that any Stein domain embeds symplectically in a smooth projective manifold $P$ with ample canonical bundle. Moreover $N$ is contained in the complement of a hyperplane section $H$, which is of course hyperplane section-like for the underlying complex structure. Up to shrinking $\Sigma$ via the Liouville flow, we can thus assume that we have a contact embedding $\Sigma \subset N \times D^{2} \subset P \times S^{2}$, where $P$ carries an integral symplectic form $\omega_{P}$, the symplectic form $\sigma$ on $S^{2}$ is normalized by $[\sigma]\left[S^{2}\right]=1$, and the image of $\Sigma$ is contained in $(P \backslash H) \times S^{2}$, where $H$ is a hyperplane section-like symplectic submanifold. We may now apply Proposition 2.11 and this concludes our proof.

Remark 2.13. Of course the condition that $H_{j}(\Sigma) \rightarrow H_{j}(W)$ is onto is equivalent to the claim that $H^{j}(W) \rightarrow H^{j}(\Sigma)$ is injective, or that $H_{j}(W) \rightarrow H_{j}(W, \Sigma)$ vanishes.

The case when $\Sigma$ is a sphere leads to the following variant of the EliashbergFloer-McDuff theorem ([McD]): the assumptions that we impose are weaker, but so is the conclusion.

Corollary 2.14. Let $(\Sigma, \xi)$ be a simply connected contact manifold admitting an embedding in a subcritical Stein manifold, and assume that $\Sigma$ is a homology sphere (resp. rational homology sphere). Any symplectically aspherical filling of $\Sigma$ is then a homology ball (resp. rational homology ball).

Proof. Indeed apply Theorem 2.6 to the case where $H_{j}(\Sigma)=0$. We conclude that $H_{j}(W)=0$.

(Counter-)examples are given by Brieskorn spheres (see Corollary 6.4). Note that if $(\Sigma, \xi)$ is the standard contact sphere, it has an obvious embedding in $\mathbb{R}^{2 n}$. In this situation, using an argument by Eliashberg, it is proved in [McD] that $W$ is simply connected. Thus we get, using Smale's $h$-cobordism theorem ([Sm]) that $W$ is diffeomorphic to the ball. This is the original Eliashberg-Floer-McDuff theorem. 


\section{The case of $\left(\mathbb{R}^{2 n}, \sigma_{0}\right)$}

In this section we denote $b_{p}(X)$ the Betti numbers of a manifold $X$ with coefficients in a given field. Thus $b_{p}(X)$ is the rank of the $p$-th homology/cohomology group.

Theorem 3.1. Assume $(\Sigma, \xi)$ admits a contact embedding in $\left(\mathbb{R}^{2 n}, \sigma_{0}\right)$, with interior component $Z$. Let $(W, \omega)$ be a symplectic filling of $(\Sigma, \xi)$ such that $W$ is symplectically aspherical and one of the following conditions is satisfied:

(a) $H_{2}(W, \Sigma)=0$.

(b) The maps $\pi_{1}(\Sigma) \rightarrow \pi_{1}(W)$ and $\pi_{1}(\Sigma) \rightarrow \pi_{1}\left(\mathbb{R}^{2 n} \backslash Z\right)$ are injective.

(c) $(\Sigma, \xi)$ is of restricted contact type in $(W, \omega)$ and in $\left(\mathbb{R}^{2 n}, \sigma_{0}\right)$.

\section{Then}

(1) any two symplectically aspherical fillings of $(\Sigma, \xi)$ which satisfy either of the conditions (a)-(c) have the same Betti numbers;

(2) given a symplectically aspherical filling $W$ which satisfies one of the conditions (a)-(c), the inclusion of $\Sigma$ in $W$ induces an injection in cohomology

$$
H^{p}(W) \longrightarrow H^{p}(\Sigma) \text {. }
$$

Moreover, we have

$$
b_{p}(\Sigma)=b_{p}(W)+b_{2 n-p-1}(W) .
$$

Remark 3.2. Condition (a) holds if $W$ is Stein and $n \geq 3$. Condition (b) holds if $\Sigma$ is simply connected. The embedding $\Sigma \hookrightarrow \mathbb{R}^{2 n}$ is always separating since $H_{2 n-1}\left(\mathbb{R}^{2 n} ; \mathbb{Z}\right)=0$.

Remark 3.3. The first statement in (2) follows from the previous section if either $\Sigma$ is simply connected or condition (a) is satisfied. The reason why we can allow more general assumptions in the case of $\mathbb{R}^{2 n}$ is that the geometry at infinity is perfectly controlled, unlike for an arbitrary subcritical Stein manifold.

Remark 3.4. As will be clear from the proof, the conclusions of Theorem 3.1 hold under the more general assumption that $\left(\mathbb{R}^{2 n} \backslash Z\right) \sqcup_{\Sigma} W$ is symplectically aspherical.

Remark 3.5. There is a natural way to endow the smooth manifold $U:=\left(\mathbb{R}^{2 n} \backslash\right.$ Z) $\sqcup_{\Sigma} W$ with a symplectic form, which coincides with $\sigma_{0}$ on $\mathbb{R}^{2 n} \backslash Z$. The assumption that $U$ is symplectically aspherical in the statement of Theorem 3.1 is understood with respect to this symplectic form. The construction is the following. Let $\alpha_{0}$ be the contact form induced on $\Sigma$ as the concave boundary of $\mathbb{R}^{2 n} \backslash Z$, and let $\alpha_{W}$ be the contact form induced on $\Sigma$ as the convex boundary of $W$. If $\alpha_{0}=\alpha_{W}$, then 
$\sigma_{0}$ and $\omega$ can be glued into a symplectic form on $U$. If $\alpha_{0}=f \alpha_{W}$ for some function $f: \Sigma \rightarrow(0, \infty)$, we reduce to the case of equality as follows. Let $m=\max _{\Sigma}(1 / f)$ and choose $R>m \max _{\Sigma}(f)$. We attach to $W$ along its boundary the finite piece of symplectization $\left([1, R] \times \Sigma, d\left(r \alpha_{W}\right)\right)$, remove what lies beyond the graph of $m f$, and denote the resulting domain by $W^{\prime}$. Then $W^{\prime}$ is diffeomorphic to $W$, it carries a natural symplectic form $\omega^{\prime}$, while its contact boundary is naturally identified with $\Sigma$ and carries the contact form $m f \alpha_{W}=m \alpha_{0}$. Up to replacing $(W, \omega)$ with $\left(W^{\prime}, \frac{1}{m} \omega^{\prime}\right)$, we can therefore assume that $\alpha_{W}=\alpha_{0}$, and the two symplectic forms on $W$ and $\mathbb{R}^{2 n} \backslash Z$ can be glued into a symplectic form on $U$.

Proof. We first show that any of the conditions (a)-(c) guarantees that the symplectic form $\omega^{\prime}$ on $U:=\left(\mathbb{R}^{2 n} \backslash Z\right) \sqcup_{\Sigma} W$ described in Remark 3.5 vanishes on spheres. Since $\omega^{\prime}=\sigma_{0}$ outside a compact set, we obtain that $U$ is diffeomorphic to $\mathbb{R}^{2 n}$ by the Eliashberg-Floer-McDuff theorem. (Although for the convenience of the formulation we use the diffeomorphism statement in the Eliashberg-Floer-McDuff theorem, we only need the fact that $U$ has the homology of a point.)

Let $C$ be a 2 -sphere in $U$, assume without loss of generality that it intersects $\Sigma$ transversally, and denote by $C_{1}$ and $C_{2}$ the pieces contained in $W$ and $\mathbb{R}^{2 n} \backslash \Sigma$ respectively.

- Let us assume (a). Then we find a cycle $\Gamma$ in $\Sigma$ such that $\partial C_{1}=\partial \Gamma$. Since $C_{1} \cup \Gamma$ is a cycle in $H_{2}(W)$ and the map $H_{2}(\Sigma) \rightarrow H_{2}(W)$ is onto, we obtain that $C_{1} \cup \Gamma$ is homologous to a cycle $C_{3}$ contained in $\Sigma$. Since $\omega$ is exact near $\Sigma$, the area of $C_{3}$ is zero and therefore the areas of $C_{1}$ and of $\Gamma$ are equal. Hence $\Gamma \cup C_{2}$ is a cycle in $\mathbb{R}^{2 n} \backslash Z$ with the same area as $C$. But the area of $\Gamma \cup C_{2}$ is zero because $\sigma_{0}$ is exact, and so is the area of $C$.

- Let us assume (b). At least one of the components of $C_{1}$ or $C_{2}$ is a disc, with boundary on $\Sigma$. By assumption, we can cap it by a disc in $\Sigma$ to get a sphere in $W$ or in $\mathbb{R}^{2 n} \backslash Z$ which, by symplectic asphericity of $W$ and $\mathbb{R}^{2 n} \backslash Z$, has zero area. We can thus inductively remove each component of $C_{1}, C_{2}$ and finally prove that $C$ has zero area.

- Let us assume (c). In this case the symplectic form on $U$ is exact.

We thus have that $U$ is diffeomorphic to $\mathbb{R}^{2 n}$. Since $\Sigma$ is contained in some large ball, denoted $B$, we equivalently have that $(B \backslash Z) \sqcup_{\Sigma} W$ is diffeomorphic to $B$. In this case the cohomology Mayer-Vietoris exact sequence is as follows:

$$
\longrightarrow H^{p}(B) \longrightarrow H^{p}(W) \oplus H^{p}(B \backslash Z) \longrightarrow H^{p}(\Sigma) \longrightarrow H^{p+1}(B) \longrightarrow .
$$

Since $H^{p}(B)=0$ for $p>0$, we see that the map

$$
H^{p}(W) \oplus H^{p}(B \backslash Z) \longrightarrow H^{p}(\Sigma)
$$

is an isomorphism for $p \geq 1$. Since it is induced by the inclusion maps, the first claim in (2) follows. 
For $p>0$ we have

$$
b_{p}(\Sigma)=b_{p}(W)+b_{p}(B \backslash Z) .
$$

Moreover, according to Alexander duality (see [G-Ha], theorem 27.5, p. 233) we have

$$
b_{p}(B \backslash Z)=b_{2 n-p-1}(Z)
$$

for $0<p<2 n-1$, which implies that, in this range, we have

$$
b_{p}(\Sigma)=b_{p}(W)+b_{2 n-p-1}(Z) .
$$

Of course, this also holds when we replace $W$ by $Z$, so that

$$
b_{p}(\Sigma)-b_{p}(Z)=b_{2 n-p-1}(Z)
$$

and finally

$$
b_{p}(\Sigma)=b_{p}(W)+\left(b_{p}(\Sigma)-b_{p}(Z)\right) .
$$

This implies $b_{p}(W)=b_{p}(Z)$ for $0<p<2 n-1$.

For $p=2 n-1$, if $B(\varepsilon)$ is a small ball inside $Z$, the inclusions

$$
B \backslash B(\varepsilon) \supset B \backslash Z \supset S^{2 n-1}
$$

imply that $b_{2 n-1}(B-Z) \geq 1$, and the exact sequence

$$
0 \longrightarrow H^{2 n-1}(W) \oplus H^{2 n-1}(B \backslash Z) \longrightarrow H^{2 n-1}(\Sigma) \longrightarrow 0
$$

implies that $b_{2 n-1}(B \backslash Z)=1$ and $b_{2 n-1}(W)=b_{2 n-1}(Z)=0$. Finally, it is clear that the equality still holds for $p=0$, since $b_{0}(\Sigma)=b_{0}(W)=1$.

Corollary 3.6. Assume $(\Sigma, \xi)$ has a Stein filling $(W, \omega)$ and has a contact embedding in $\left(\mathbb{R}^{2 n}, \sigma_{0}\right), n \geq 3$. Then

$$
\left\{\begin{array}{l}
b_{p}(\Sigma)=b_{p}(W) \text { for } 0 \leq p \leq n-2, \\
b_{n-1}(\Sigma)=b_{n}(\Sigma)=b_{n}(W)+b_{n-1}(W) .
\end{array}\right.
$$

Thus the homology of $W$ is completely determined by the homology of $\Sigma$ except, maybe, in degree $n-1$ and $n$. It is completely determined by the homology of $\Sigma$ if $b_{n}(\Sigma)=0$ or $W$ is subcritical Stein.

Proof. The assumptions of Theorem 3.1 are satisfied, since $H_{2}(W, \Sigma)=H^{2 n-2}(W)$ vanishes if $n \geq 3$. It follows that $b_{p}(W)$ is determined by $b_{p}(\Sigma)$, except maybe in dimensions $n-1$ and $n$. If $b_{n}(\Sigma)=0$ we obtain $b_{n}(W)=b_{n-1}(W)=0$, and if $W$ is subcritical we have $b_{n}(W)=0$ and therefore $b_{n-1}(\Sigma)=b_{n-1}(W)$. 
Remark 3.7. Mei-Lin Yau proved (see [Yau]) that, if $W$ is subcritical Stein and the first Chern class of the complex vector bundle defined by $\xi$ vanishes, then cylindrical contact homology in the trivial homotopy class $H C_{*}^{0}(\Sigma, \alpha)$ is well-defined for a suitably chosen contact form $\alpha$, and we have

$$
H C_{*}^{0}(\Sigma, \alpha) \simeq H_{*}(W, \Sigma) \otimes H_{*}\left(\mathbb{C} P^{\infty}\right)[2] .
$$

By definition, the chain complex underlying $H C_{*}^{0}(\Sigma, \alpha)$ is generated by contractible closed Reeb orbits for the contact form $\alpha$. The degree of a generator $\gamma$ is defined to be $C Z(\gamma)+n-3$, where $C Z(\gamma)$ denotes the Conley-Zehnder index of the linearized Reeb flow along $\gamma$ in the transverse direction, computed with respect to a trivialization of $\xi$ along $\gamma$ induced by a trivialization over a spanning disc. The symbol [2] denotes a shift in degree by 2 .

It is therefore a general fact that the homology of a subcritical filling is determined by the contact structure $(\Sigma, \xi)$. It is however not clear whether in general it is already determined by the knowledge of the topology of $\Sigma$ (i.e. independently from $\xi$ or the topology of a filling).

As a first consequence of Corollary 3.6 and Mei-Lin Yau's result we have:

Corollary 3.8. Assume $(\Sigma, \xi)$ satisfies $c_{1}(\xi)=0$, has a subcritical Stein filling $(W, \omega)$ and has a contact embedding in $\left(\mathbb{R}^{2 n}, \sigma_{0}\right)$. Then the rank of $H C_{*}^{0}(\Sigma, \alpha)$ is determined by $H_{*}(\Sigma)$. Indeed, we have

$$
\operatorname{rank}\left(H C_{k}^{0}(\Sigma, \alpha)\right)=\sum_{\substack{2 n-2-k \leq p \leq n-1 \\ p \equiv k \bmod 2}} b_{p}(\Sigma) .
$$

Proof. Note that assumption (a) from Theorem 3.1 is automatically satisfied: we are in the Stein case. The result is a straightforward application of Corollary 3.6, Mei-Lin Yau's theorem and the duality $H^{2 n-k}(W) \simeq H_{k}(W, \partial W)$.

Thus

$$
H C_{k}^{0}(\Sigma, \alpha)=\bigoplus_{m \geq 0} H_{k-2 m+2}(W, \Sigma)=\bigoplus_{m \geq 0} H^{2 n-2-k+2 m}(W)
$$

and $b_{2 n-2-k+2 m}(W)=b_{2 n-2-k+2 m}(\Sigma)$ for $0 \leq 2 n-2-k+2 m \leq n-1$. Setting $p=2 n-2-k+2 m$ yields the above formula.

To state the next application of our theorem, let us recall the following definitions. A Hermitian line bundle $\mathscr{L} \stackrel{\pi}{\rightarrow} N$ over a symplectic manifold $\left(N^{2 n-2}, \beta\right)$ is called negative if $c_{1}(\mathscr{L})=-[\beta]$. Equivalently, there exists a Hermitian connection $\nabla$ whose curvature satisfies $\frac{1}{2 i \pi} F^{\nabla}=-\beta$. Such a connection determines the transgression 1 form $\theta^{\nabla} \in \Omega^{1}\left(\mathscr{L} \backslash 0_{\mathscr{L}}, \mathbb{R}\right)$ which, by definition, vanishes on the horizontal distribution 
and is equal to $\frac{1}{2 \pi}$ times the angular form in the fibers. Denoting $r(u):=|u|$, the total space $\mathscr{L}$ carries the symplectic form $\omega:=\pi^{*} \beta+d\left(r^{2} \theta^{\nabla}\right)$, which is exact on $\mathscr{L} \backslash 0_{\mathscr{L}}$ with $\omega=d\left(\left(1+r^{2}\right) \theta^{\nabla}\right)$. The unit disc bundle $W=\{u \in \mathscr{L}:|u| \leq 1\}$ is a symplectic manifold with contact type boundary. For details we refer to [O], Section 3.3.

Proposition 3.9. Let $(\Sigma, \xi)$ be the contact boundary of the unit disc bundle $(W, \omega)$ associated to a negative line bundle over $\left(N^{2 n-2}, \beta\right)$. Assume that $(N, \beta)$ is symplectically aspherical. Then $(\Sigma, \xi)$ has no contact embedding in $\left(\mathbb{R}^{2 n}, \sigma_{0}\right)$ with interior $Z$, such that $\left(\mathbb{R}^{2 n} \backslash Z\right) \sqcup_{\Sigma} W$ is symplectically aspherical. The same holds for $n \geq 3$ and for any contact manifold obtained by contact surgery (as in [E], [W]) of index $k$ for any $k \in[3, n]$.

Proof. The Gysin exact sequence is

$$
\longrightarrow H^{p-2}(N) \stackrel{\beta \cup}{\longrightarrow} H^{p}(N) \longrightarrow H^{p}(\Sigma) \longrightarrow H^{p-1}(N) \longrightarrow
$$

and, in degree 2 , we get

$$
H^{2}(\Sigma)=H^{2}(N) /\langle[\beta]\rangle \oplus \operatorname{ker}\left([\beta] \cup: H^{1}(N) \longrightarrow H^{3}(N)\right) .
$$

Hence

$$
b_{2}(\Sigma)<b_{2}(N)+b_{1}(N)=b_{2}(N)+b_{2 n-2-1}(N)=b_{2}(W)+b_{2 n-2-1}(W),
$$

and this contradicts Theorem 3.1.

Let us now see what happens when we make a contact surgery. We shall denote our hypersurface by $\Sigma^{-}, W^{-}$will be its filling, and $\Sigma^{+}$will be the result of the surgery on $\Sigma^{-}$along a $(k-1)$-dimensional isotropic sphere. Let us denote by $A_{k} \simeq D^{k} \times D^{2 n-k}$ the attached handle, and denote $\partial^{-} A_{k}=S^{k-1} \times D^{2 n-k}, \partial^{+} A_{k}=D^{k} \times S^{2 n-k-1}$, so that the new filling of $\Sigma^{+}$is $W^{+}=W^{-} \cup_{\partial-} A_{k} A_{k}$. We first need to prove that $W^{+}$is symplectically aspherical. But the homotopy exact sequence of the pair $\left(W^{+}, W^{-}\right)$ is given by

$$
\longrightarrow \pi_{3}\left(W^{+}, W^{-}\right) \longrightarrow \pi_{2}\left(W^{-}\right) \longrightarrow \pi_{2}\left(W^{+}\right) \longrightarrow \pi_{2}\left(W^{+}, W^{-}\right) \longrightarrow
$$

and $\pi_{2}\left(W^{+}, W^{-}\right) \simeq \pi_{2}\left(A_{k}, \partial^{-} A_{k}\right) \simeq \pi_{2}\left(D^{k}, \partial D^{k}\right)=0$ for $k \geq 3$. Thus the inclusion of $W^{-}$in $W^{+}$induces a surjective map on $\pi_{2}$, hence if $[\omega] \pi_{2}\left(W^{-}\right)=0$, we also have $[\omega] \pi_{2}\left(W^{+}\right)=0$.

Let us now first consider the case $k \geq 4$. We claim that we have $b_{2}\left(\Sigma^{+}\right)=$ $b_{2}\left(\Sigma^{-}\right)$and $b_{2}\left(W^{+}\right)=b_{2}\left(W^{-}\right)$. Indeed the homology exact sequence for the pair $\left(W^{+}, W^{-}\right)$is

$$
\longrightarrow H_{3}\left(W^{+}, W^{-}\right) \longrightarrow H_{2}\left(W^{-}\right) \longrightarrow H_{2}\left(W^{+}\right) \longrightarrow H_{2}\left(W^{+}, W^{-}\right) \longrightarrow
$$


but $H_{j}\left(W^{+}, W^{-}\right) \simeq H_{j}\left(A_{k}, \partial^{-} A_{k}\right) \simeq H_{j}\left(D^{k}, \partial D^{k}\right)=0$ for $j=2,3$ and $k \geq 4$, so $b_{2}\left(W^{+}\right)=b_{2}\left(W^{-}\right)$.

Similarly the Mayer-Vietoris exact sequence for $\Sigma^{ \pm}=\Sigma^{-} \backslash\left(\partial^{-} A_{k}\right) \cup \partial^{ \pm} A_{k}$ reads

$$
\begin{aligned}
H_{2}\left(S^{k-1} \times S^{2 n-k-1}\right) & \longrightarrow H_{2}\left(\Sigma^{-} \backslash \partial^{-} A_{k}\right) \oplus H_{2}\left(\partial^{ \pm} A_{k}\right) \\
& \longrightarrow H_{2}\left(\Sigma^{ \pm}\right) \longrightarrow H_{1}\left(S^{k-1} \times S^{2 n-k-1}\right)=0 .
\end{aligned}
$$

When $k \geq 4$, the groups $H_{2}\left(S^{k-1} \times S^{2 n-k-1}\right)$ and $H_{2}\left(\partial^{ \pm} A_{k}\right)$ vanish, so that we have isomorphisms

$$
H_{2}\left(\Sigma^{-} \backslash \partial^{-} A_{k}\right) \simeq H_{2}\left(\Sigma^{ \pm}\right)
$$

and therefore $b_{2}\left(\Sigma^{+}\right)=b_{2}\left(\Sigma^{-}\right)$.

Let us now deal with the case $k=3, n \geq 4$. In the "-" case, the first map in the exact sequence (3.1) is injective (since its projection on the second summand is induced by the inclusion $\left.S^{2} \times S^{2 n-4} \rightarrow S^{2} \times D^{2 n-3}\right)$. Since $2 n-4>2$, we obtain $b_{2}\left(\Sigma^{-}\right)=b_{2}\left(\Sigma^{-} \backslash \partial^{-} A_{3}\right)$. In the "+" case, we have $H_{2}\left(\partial^{+} A_{3}\right)=0$ and $b_{2}\left(\Sigma^{+}\right) \leq b_{2}\left(\Sigma^{-} \backslash \partial^{-} A_{3}\right)=b_{2}\left(\Sigma^{-}\right)$.

Below we write down the homology exact sequences of the pairs $\left(W^{+}, W^{-}\right)$and $\left(\Sigma^{+}, \Sigma^{+} \cap W^{-}\right)$:

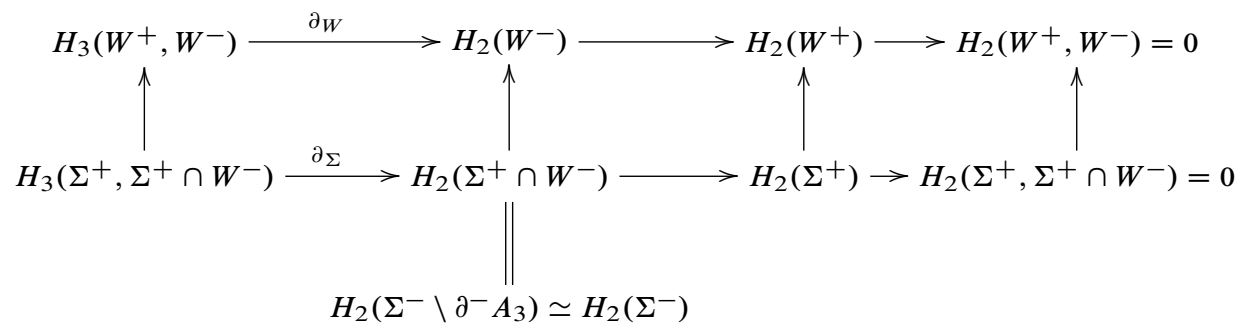

The left-hand side vertical map is an isomorphism since

$$
\begin{aligned}
H_{3}\left(\Sigma^{+}, \Sigma^{+} \cap W^{-}\right) \simeq H_{3}\left(D^{3} \times S^{2 n-4}, S^{2} \times S^{2 n-4}\right) \\
\quad \stackrel{\simeq}{\longrightarrow} H_{3}\left(D^{3} \times D^{2 n-3}, S^{2} \times D^{2 n-3}\right) \simeq H_{3}\left(W^{+}, W^{-}\right)
\end{aligned}
$$

Hence either the map $\partial_{W}$ is injective, and thus so is $\partial_{\Sigma}$ and consequently $b_{2}\left(W^{+}\right)=$ $b_{2}\left(W^{-}\right)-1$ and $b_{2}\left(\Sigma^{+}\right)=b_{2}\left(\Sigma^{-}\right)-1$, or it is zero, and then $b_{2}\left(W^{+}\right)=b_{2}\left(W^{-}\right)$ and $b_{2}\left(\Sigma^{+}\right) \leq b_{2}\left(\Sigma^{-}\right)$.

For $n=k=3$, we leave it to the reader to check that

$$
H_{2}\left(\Sigma^{-}\right) \simeq H_{2}\left(\Sigma^{-} \backslash \partial^{-} A_{3}\right) / \operatorname{Im}\left(H_{2}\left(\{\mathrm{pt}\} \times S^{2}\right)\right)
$$

and

$$
H_{2}\left(\Sigma^{+}\right) \simeq H_{2}\left(\Sigma^{-} \backslash \partial^{-} A_{3}\right) / \operatorname{Im}\left(H_{2}\left(S^{2} \times S^{2}\right)\right)
$$


so that $b_{2}\left(\Sigma^{+}\right)$equals either $b_{2}\left(\Sigma^{-}\right)$or $b_{2}\left(\Sigma^{-}\right)-1$. Again using the same argument as above, whenever $b_{2}\left(W^{+}\right)=b_{2}\left(W^{-}\right)-1$ we have $b_{2}\left(\Sigma^{+}\right)=b_{2}\left(\Sigma^{-}\right)-1$. This concludes our proof.

Remark 3.10. According to [La], if $(\Sigma, \xi)$ has a contact embedding in $\mathbb{R}^{2 n}$, the same holds for any manifold obtained by contact surgery over an isotropic sphere of dimension $\leq n-1$. In contrast, we display here an obstruction to embedding $\Sigma$ in $\mathbb{R}^{2 n}$ that survives such a surgery.

Examples 3.11. The symplectic asphericity condition in Proposition 3.9 is necessary: the manifold $\left(\mathbb{C} P^{n-1}, \sigma_{0}\right)$ is not symplectically aspherical, and $\left(S^{2 n-1}, \alpha_{0}\right)$ has a contact embedding into $\left(\mathbb{R}^{2 n}, \sigma_{0}\right)$.

Remark 3.12. The previous proof does not generalize to higher rank bundles. Let us call a Hermitian vector bundle $\varepsilon \stackrel{\pi}{\rightarrow} N$ over a symplectic manifold $\left(N^{2 n-2}, \beta\right) n e g$ ative if it admits a Hermitian connection $\nabla$ whose curvature $\frac{1}{i} F^{\nabla} \in \Omega^{2}(N$, End $\mathcal{E})$ is negative definite. This means that, for any $\beta$-compatible almost complex structure $J$ on the base $N$ and any non-zero vector $v \in T N$, we have $\frac{1}{i} F^{\nabla}(v, J v)<0$.

Let $\mathbb{P}(\mathcal{E})$ denote the projectivized bundle and $\mathscr{L}_{\mathcal{E}} \stackrel{\pi}{\rightarrow} \mathbb{P}(\mathscr{E})$ be the tautological line bundle. Then $\mathscr{L}_{\mathcal{E}}$ is a negative Hermitian line bundle, and the total space carries the symplectic form $\omega_{\mathscr{L}}=\pi^{*} \omega_{F S}+\Omega_{\mathscr{L}}$, where $\omega_{F S}$ is the curvature form on $\mathbb{P}(\mathcal{E})$ and $\Omega_{\mathscr{L}}=d\left(r^{2} \theta^{\nabla}\right)$, with $r(u)=|u|$ and $\theta^{\nabla}$ the transgression 1-form (see the preamble to Proposition 3.9). Denoting $W_{\mathscr{L}}=\left\{u \in \mathscr{L}_{\mathcal{E}}:|u| \leq 1\right\}$, we see that $\Sigma=\partial W_{\mathscr{L}}$ is a contact manifold. However, the filling $W_{\mathscr{L}}$ is not symplectically aspherical since it contains $\mathbb{P}(\mathcal{E})$ as a symplectic submanifold.

The manifold $\Sigma$ can also be realized inside $\mathcal{E}$ as $\{u \in \mathcal{E}:|u|=1\}$, via a natural diffeomorphism $\mathscr{L}_{\mathcal{E}} \backslash 0_{\mathscr{L}_{\mathcal{E}}} \simeq \mathcal{E} \backslash 0_{\mathcal{E}}$. This diffeomorphism transforms $W_{\mathscr{L}}$ into $W=\{u \in \mathcal{E}:|u| \leq 1\}$. The pull-back of $\Omega_{\mathscr{L}}$ via this diffeomorphism, denoted $\Omega$, is symplectic on $\mathcal{E} \backslash 0_{\mathcal{E}}$ and extends over $0_{\mathcal{E}}$, as equal to the area form in the fibers and vanishing along the zero-section. We can thus equip $\varepsilon$ with the symplectic form $\omega=\pi^{*} \beta+\Omega$. If $\beta$ is symplectically aspherical, then so is $\omega$. However, $\Sigma=\partial W$ is not of contact type since the restriction of $\pi^{*} \beta$ to $\Sigma$ is not exact for $r \geq 2$, as shown by the Gysin exact sequence.

The outcome of this discussion is that, even if $(N, \beta)$ is symplectically aspherical, $\Sigma$ does not appear naturally as contact type boundary of a symplectically aspherical manifold. We feel that a result analogous to Proposition 3.9 should hold for higher rank negative vector bundles, but our methods do not apply in this case.

For the details of the above constructions we refer to [O], Section 3.4.

Proposition 3.13. Let $L$ be a compact manifold admitting a Lagrangian embedding into $\mathbb{R}^{2 n}$ and $n \geq 3$. Then any symplectically aspherical filling $W$ of $S T^{*} L$ such 
that $H_{2}\left(W, S T^{*} L\right)=0$ has the same homology as $D T^{*} L$ (and hence the homology of $L)$.

Proof. Indeed, the hypothesis implies that $S T^{*} L$ has a contact (non exact !) embedding into $\mathbb{R}^{2 n}$, so that we can apply Theorem 3.1. Using Thom's isomorphism, the condition $\mathrm{H}_{2}\left(D T^{*} L, S T^{*} L\right)=0$ is clearly satisfied.

Let now $S T^{*} L$ be the unit cotangent bundle of $L$. The spectral sequence of this sphere bundle yields the following dichotomy:

- either the Euler class vanishes, and then

$$
b_{p}\left(S T^{*} L\right)=b_{p}(L)+b_{p-(n-1)}(L),
$$

- or the Euler class is non zero and then

$$
\left\{\begin{array}{l}
b_{p}\left(S T^{*} L\right)=b_{p}(L)+b_{p-(n-1)}(L) \text { for } p \neq n-1, n \\
b_{n}\left(S T^{*} L\right)=b_{n-1}\left(S T^{*} L\right)=b_{n-1}(L)=b_{1}(L) .
\end{array}\right.
$$

The formula

$$
b_{p}(\Sigma)=b_{p}(W)+b_{2 n-p-1}(W)
$$

becomes

(a) in the first case

$$
b_{p}(L)+b_{p-(n-1)}(L)=b_{p}(L)+b_{2 n-p-1}(L),
$$

hence

$$
b_{p-(n-1)}(L)=b_{2 n-p-1}(L),
$$

that is, the Poincaré duality formula;

(b) in the second case

$$
b_{1}(L)=b_{n-1}(L)=b_{n}(L)+b_{2 n-n-1}(L)=b_{n}(L)+b_{n-1}(L) .
$$

This implies $b_{n}(L)=0$, which is impossible (at least for orientable $L$ ).

Proposition 3.14. Let $L$ be an orientable manifold with non zero Euler class. Then $S T^{*} L$ has no contact embedding in $\mathbb{R}^{2 n}, n \geq 3$. The same holds for any contact manifold obtained from such a $S T^{*} L$ by surgery of index $3 \leq k \leq n-3$.

Proof. The case of $S T^{*} L$ has been already proved above. The surgery does not modify the conditions $H_{2}(W, \Sigma)=0$ nor does it change $b_{n}(\Sigma)$ or $b_{n}(W), b_{n-1}(W)$. This concludes our proof.

Remark 3.15. The condition $e(L)=0$ is exactly the condition needed to be able to find a Lagrangian immersion of $L$ regularly homotopic to an embedding. We however suspect that there are no embeddings of $S T^{*} L$ as a smooth hypersurface in $\mathbb{R}^{2 n}$. 


\section{The Stein subcritical case}

In this section we assume that $\left(\Sigma_{1}, \xi_{1}\right)$ has a separating contact embedding in a subcritical Stein domain $\left(W_{2}, \omega_{2}\right)$ with boundary $\left(\Sigma_{2}, \xi_{2}\right)$, and we denote by $V_{1}$ the bounded component of $W_{2} \backslash \Sigma_{1}$. We denote by $\left(W_{1}, \omega_{1}\right)$ an arbitrary symplectically aspherical filling of $\left(\Sigma_{1}, \xi_{1}\right)$ such that one of the following assumptions holds (cf. Theorem 2.6):

- $H_{2}\left(W_{1}, \Sigma_{1}\right)=0$;

- $\Sigma_{1}$ is simply connected.

Proposition 4.1. Under the above assumptions, we have that

$$
b_{j}\left(W_{1}\right) \leq b_{j}\left(\Sigma_{1}\right)+\min \left(0, b_{j}\left(\Sigma_{2}\right)-b_{j}\left(W_{2} \backslash V_{1}\right)\right) .
$$

Proof. Note that given an exact sequence $A \stackrel{f}{\rightarrow} B \stackrel{g}{\rightarrow} C$ we have $\operatorname{dim}(B)=$ $\operatorname{dim}(\operatorname{ker}(g))+\operatorname{dim}(\operatorname{Im}(g))=\operatorname{dim}(\operatorname{Im} f)+\operatorname{dim}(\operatorname{Im}(g)) \leq \operatorname{dim}(A)+\operatorname{dim}(C)$. Using the Mayer-Vietoris exact sequence of $\left(W_{2} \backslash V_{1}\right) \sqcup W_{1}$ and the inequality $\operatorname{dim} H_{j}\left(\Sigma_{2}\right) \geq \operatorname{dim} H_{j}\left(\left(W_{2} \backslash V_{1}\right) \sqcup W_{1}\right)$, we get that

$$
b_{j}\left(W_{2} \backslash V_{1}\right)+b_{j}\left(W_{1}\right) \leq b_{j}\left(\Sigma_{2}\right)+b_{j}\left(\Sigma_{1}\right) .
$$

Thus

$$
b_{j}\left(W_{1}\right) \leq\left(b_{j}\left(\Sigma_{2}\right)-b_{j}\left(W_{2} \backslash V_{1}\right)\right)+b_{j}\left(\Sigma_{1}\right) .
$$

According to Theorem 2.6 we have $b_{j}\left(W_{1}\right) \leq b_{j}\left(\Sigma_{1}\right)$, and our claim follows.

Note that $b_{j}\left(W_{2} \backslash V_{1}\right)=b_{2 n-j}\left(W_{2}, V_{1} \cup \Sigma_{2}\right)$ by Poincaré duality and excision. Note also that the above result is stronger than Theorem 2.6 only when $b_{j}\left(\Sigma_{2}\right)-$ $b_{j}\left(W_{2} \backslash V_{1}\right)<0$. This happens for example if $\Sigma_{2}$ is a homology sphere.

The first part of the following result has been obtained in a weaker form and by different methods in [C-F-O] (see also proposition 5.12).

Proposition 4.2. Let $L$ be an orientable closed manifold of dimension $\geq 3$, with non-zero Euler class. Then $S T^{*} L$ has no contact embedding in a subcritical Stein manifold. As before, this also holds for any manifold obtained from $S T^{*} L$ by contact surgery of index $k \in[3, n-1]$.

Proof. Since $n \geq 3$, the group $H_{2}\left(D T^{*} L, S T^{*} L\right)$ is zero, so assumption (a) of Theorem 2.6 is satisfied. The Gysin exact sequence of $S T^{*} L$ shows that the map $H_{n}\left(S T^{*} L\right) \rightarrow H_{n}(L)$ vanishes. This contradicts Theorem 2.6. The case of manifolds obtained by surgery is dealt with as in Proposition 3.9. 


\section{Obstructions from symplectic homology}

In this section we assume that $(\Sigma, \xi)$ is a contact manifold whose first Chern class $c_{1}(\xi)$ vanishes. All the symplectic fillings $(W, \omega)$ of $(\Sigma, \xi)$ that we consider are assumed to satisfy $c_{1}(T W)=0$.

Definition 5.1. Let $(W, \omega)$ be a connected symplectically aspherical manifold with contact type boundary. We say that $(W, \omega)$ is an $S A W C$ manifold if $S H_{*}(W, \omega)=0$.

Remark 5.2. The vanishing of $S H_{*}(W)$ is equivalent to the fact that $W$ satisfies the Strong Algebraic Weinstein Conjecture as defined in [V], stating that the canonical map $H_{2 n}(W, \partial W) \rightarrow S H_{n}(W)$ is not injective. This follows from the following three observations: (i) non-injectivity of the map $H_{2 n}(W, \partial W) \rightarrow S H_{n}(W)$ is equivalent to its vanishing, since $H_{2 n}(W, \partial W)$ is 1-dimensional; (ii) symplectic homology is a ring with unit $[\mathrm{McL}]$, and the unit is the image of the fundamental class of $W$ under the map $H_{2 n}(W, \partial W) \rightarrow S H_{n}(W)$ [Se]; (iii) vanishing of the unit for $S H_{*}(W)$ is equivalent to the vanishing of $S H_{*}(W)$.

It is proved in [B-O-2], Corollary 1.4 that an SAWC manifold also satisfies the Equivariant Algebraic Weinstein Conjecture from [V]. This can also be seen using the spectral sequence connecting the usual version of symplectic homology to the equivariant version $[\mathrm{V}]$.

If we have an exact embedding $\left(V_{1}, \omega_{1}\right)$ into $\left(W_{1}, \omega_{1}\right)$, there is an induced transfer map (see $[\mathrm{V}])$

$$
S H_{*}\left(W_{1}\right) \longrightarrow S H_{*}\left(V_{1}\right)
$$

which, according to Mark McLean (see [McL]), is a unital ring homomorphism. This implies the following result:

Proposition 5.3 ([McL]). Let $(V, \omega)$ be an exact symplectic submanifold of $(W, \omega)$. If $(W, \omega)$ is SAWC then $(V, \omega)$ is also SAWC.

It is easy to find SAWC manifolds which are not Stein. For example, we have:

Proposition $5.4([\mathrm{O}])$. Let $P$ be any exact symplectic manifold with contact type boundary. Then, for any exact SAWC manifold $W$, we have that $P \times W$ is $S A W C$. Also, the total space of a negative symplectic fibration in the sense of $[\mathrm{O}]$ with fiber $W$ is $S A W C$.

Proposition 5.5 ([C2]). Let $W^{\prime}$ be obtained from $W$ by attaching handles of index $\leq n-1$. Then $S H_{*}(W) \simeq S H_{*}\left(W^{\prime}\right)$. In particular if $W$ is $S A W C$, the same holds for $W^{\prime}$.

The following statement is contained in [C-F-O], Corollary 1.15 and Remark 1.19. 
Theorem 5.6. Let $(\Sigma, \xi)$ be a contact manifold for which there exists a contact form $\alpha$ whose closed characteristics are nondegenerate and have Conley-Zehnder index strictly bigger than $3-n$. Let $i:(\Sigma, \xi) \hookrightarrow(W, \omega)$ be a separating exact embedding in an SAWC manifold $(W, \omega)$. Assume $i_{*}: \pi_{1}(\Sigma) \rightarrow \pi_{1}(W)$ is injective. Then the Betti numbers of the interior $V$ of $\Sigma$ in any coefficient field are determined by the contact structure $\xi$ (and do not depend on the choice of the SAWC manifold $W$ ).

Proof. By Proposition 5.3 we have $S H_{*}(V)=0$. The relative exact sequence in symplectic homology (see [V]) then implies that

$$
S H_{*}^{+}(V) \simeq H_{*+n-1}(V, \partial V) .
$$

We can assume without loss of generality that the contact form induced by the contact embedding on $\Sigma$ is equal to $\alpha$. Let $\widehat{V}=(V, \omega) \cup([1, \infty[\times \Sigma, d(r \alpha))$ be the symplectization of $V$, obtained by gluing a semi-infinite cone along the boundary. If the Reeb vector field associated to the contact form $\alpha$ has no closed characteristic with Conley-Zehnder index $\leq 3-n$, and if $i_{*}: \pi_{1}(\Sigma) \rightarrow \pi_{1}(V)$ is injective, then there is no rigid holomorphic plane in $\widehat{V}$ bounding a closed characteristic. In this case, it is a consequence of the stretch-of-the-neck argument in [B-O-1] that $\mathrm{SH}_{*}^{+}(\mathrm{V})$ depends only on the contact boundary $\partial V=\Sigma$ (see also [C-F-O], Corollary 1.15). As a consequence, the Betti numbers $b_{j}(V, \partial V)$ only depend on $\xi$.

Now if we have another exact embedding of $\Sigma$ in $W^{\prime}$ and $W^{\prime}$ is also SAWC, the interior $V^{\prime}$ of $\Sigma$ in $W^{\prime}$ must have the same cohomology as $V$.

Proposition 5.7. Let $(\Sigma, \xi)$ be the boundary of a subcritical Stein manifold $(W, \omega)$. Let $(M, \omega)$ be an SAWC manifold such that $(\Sigma, \xi)$ has an exact separating embedding into $(M, \omega)$, with interior $Z$. Then $H_{*}(Z) \simeq H_{*}(W)$.

Proof. First of all, by Proposition 5.3, we have $S H_{*}(Z)=0$. On the one hand the exact sequence ([V])

$$
\longrightarrow S H_{*}(Z) \longrightarrow S H_{*}^{+}(Z) \longrightarrow H_{*+n-1}(Z, \Sigma) \longrightarrow S H_{*-1}(Z) \longrightarrow
$$

shows that $H_{*}(Z, \Sigma) \simeq S H_{*+1-n}^{+}(Z)$. On the other hand, since $\Sigma$ bounds a subcritical Stein manifold, there exists a contact form $\alpha$ such that the Reeb orbits are all nondegenerate and of index $>3-n$ (cf. [Yau]), so the proof of Theorem 5.6 implies that $S H_{*}^{+}(Z) \simeq S H_{*}^{+}(W)$. This last space is in turn isomorphic to $H_{*+n-1}(W, \Sigma)$ by the same argument, and finally $H_{*}(Z, \Sigma) \simeq H_{*}(W, \Sigma)$, hence $H_{*}(Z) \simeq H_{*}(W)$.

Remark 5.8. The condition that $W$ is subcritical is not really necessary. We only need $W$ to be SAWC provided there is a contact form defining $\xi$ for which all closed Reeb orbits are nondegenerate and have index $>3-n$.

Remark 5.9. Proposition 5.7 can be compared to the following result: 
Corollary 5.10 ([Yau]). Let $W$ be a subcritical Stein manifold with boundary $\partial W$ such that $\left.c_{1}(T W)\right|_{\pi_{2}(W)}=0$. Then any subcritical Stein manifold with the same boundary $\partial W$ and whose first Chern class vanishes on the second homotopy group has the same homology as $W$.

Proof. This follows from the main computation of [Yau] (cf. Remark 3.7),

$$
H C_{*}^{0}(\partial W, \alpha) \simeq H_{*}(W, \partial W) \otimes H_{*}\left(\mathbb{C} P^{\infty}\right)[2],
$$

which implies directly that the homology of a subcritical Stein filling is determined by the contact structure of the boundary.

Remark 5.11. Note that, when $\Sigma=S^{2 n-1}$, we may apply our proposition to $W=$ $D^{2 n}$. Thus we prove that any symplectically aspherical filling $Z$ with vanishing first Chern class satisfies $H_{*}(Z)=0$ in nonzero degree. Thus, if $Z$ is simply connected and $n \geq 3$, it is diffeomorphic to a ball. This is a weak version of the Eliashberg-Floer-McDuff theorem mentioned in the previous section, but note that the above proof does not make use of it and also that it extends to many other contact manifolds.

Let us now use the above tools to find obstructions to contact embeddings. We first have:

Proposition 5.12 ([C-F-O]). If $(\Sigma, \xi)=\left(S T^{*} L\right.$, $\left.\xi_{\text {std }}\right)$ with $L$ a closed simply connected manifold, then $(\Sigma, \xi)$ has no separating exact embedding in an SAWC manifold $(M, \omega)$. Here $\xi_{\text {std }}$ denotes the standard contact structure on $S T^{*} L$.

Proof. Since the characteristic flow on $S T^{*} L$ is the geodesic flow, it has all closed trajectories of index $\geq 0>3-n$ if $n>3$ (in the cases $n=2,3$ we have that $L$ is a sphere and we can find a metric for which all closed geodesics have index $>3-n$ ). Assuming the existence of such an embedding, with interior $Z$, the proof of Theorem 5.6 shows that $S H_{*}^{+}(Z)$ depends only on the boundary $(\Sigma, \xi)$. We obtain on the one hand $S H_{*}^{+}(Z) \simeq H_{*+n-1}(Z, \partial Z)$, and on the other hand $S H_{*}^{+}(Z) \simeq$ $S H_{*}^{+}\left(D T^{*} L\right)$. But $S H_{*}^{+}\left(D T^{*} L\right) \simeq H_{*}(\Lambda L, L)$, where $\Lambda L$ denotes the free loop space of $L$. Hence $S H_{*}^{+}\left(D T^{*} L\right)$ is infinite dimensional, a contradiction.

Remark 5.13. Let $(M, \omega)$ be obtained by attaching subcritical handles to $D T^{*} L$. Provided one can prove that the Reeb orbits on $(\partial M, \xi)$ still have index $>3-n$, our argument extends to show that $(\partial M, \xi)$ has no contact embedding in an SAWC manifold.

The case of circle bundles can also be dealt with using contact and Floer homology, as follows. 
Proposition 5.14. Let $(\Sigma, \xi)$ be the unit circle bundle associated to a negative line bundle $\mathscr{L}$ over a symplectically aspherical manifold $\left(N^{2 n-2}, \beta\right)$ such that $c_{1}(T N)=$ 0 . Then, for $n \geq 2, \Sigma$ does not bound a subcritical Stein manifold with vanishing first Chern class. The same holds for any contact manifold obtained by subcritical surgery on $(\Sigma, \xi)$ of index $\neq 2,3$.

Proof. Indeed, let $M$ denote the manifold bounded by $\Sigma$. If $W$ is the unit disc bundle associated to $\Sigma$, we have $\partial W=\Sigma$ and, using that $S H_{*}(W)=0$ ([O]) and the exact sequence

$$
\longrightarrow S H_{*}(W) \longrightarrow S H_{*}^{+}(\Sigma) \longrightarrow H_{*+n-1}(W, \Sigma) \longrightarrow
$$

we obtain

$$
S H_{*}^{+}(\Sigma) \simeq H_{*+n-1}(W, \Sigma) \simeq H_{*+n-3}(N) .
$$

The same exact sequence with $M$ yields

$$
S H_{*}^{+}(\Sigma) \simeq H_{*+n-1}(M, \Sigma) \simeq H^{n-*+1}(M)
$$

But this last space vanishes for $* \leq 1$ while $H_{*+n-3}(N)$ is non-zero for $*=3-n$. When $n \geq 2$ we get a contradiction. Now since $k \neq 2,3, H_{2}(W, \Sigma)$ does not change, so remains equal to $H_{0}(N)=\mathbb{Q}$. But we must have $H_{2}(W, \Sigma)=S H_{3-n}^{+}(\Sigma)=$ $H^{2 n-2}(M)=0$. A contradiction.

Remark 5.15. This partially answers a question of Biran in [Bi] who asked the same question in the Stein case (not subcritical). A different answer was given by Popescu-Pampu in [Po]

\section{Brieskorn manifolds, McLean's examples}

We consider an isolated singularity of holomorphic germ. For example, assume we are given $V$ a complex submanifold in $\mathbb{C}^{n+1}$ with an isolated singularity at the origin. We then consider the submanifold $\Sigma_{\varepsilon}=S_{\varepsilon} \cap V$, where $S_{\varepsilon}=\left\{\left.z \in \mathbb{C}^{n+1}|| z\right|^{2}=\varepsilon\right\}$ and $\varepsilon>0$ is small enough. The maximal complex subspace of the tangent space defines a hyperplane distribution which happens to be a contact structure, and whose isotopy class is independent of $\varepsilon$. In case the singularity is smoothable, $\Sigma_{\varepsilon}$ bounds a Stein manifold $W$.

Example 6.1. If $V$ is the hypersurface $f^{-1}(0)$ where $f$ is polynomial, then the singularity is always smoothable. According to [M], the manifold $W$ is homotopy equivalent to a wedge of $n$-spheres. The number $\mu \geq 0$ of spheres is called the Milnor number of the singularity. We obtain that $W$ is $(n-1)$-connected and $H_{n}(W)=\mathbb{Z}^{\mu}$. 
The boundary $\Sigma:=\partial W$ is $(n-2)$-connected. It is called the link of the singularity. For $n \geq 2$ the long exact sequence of the pair $(W, \Sigma)$ reduces to

$$
0 \longrightarrow H_{n}(\Sigma) \longrightarrow H_{n}(W) \stackrel{S}{\longrightarrow} \operatorname{Hom}\left(H_{n}(W), \mathbb{Z}\right) \longrightarrow H_{n-1}(\Sigma) \longrightarrow 0 .
$$

Here we used the identification $H_{n}(W, \Sigma) \simeq H^{n}(W) \simeq \operatorname{Hom}\left(H_{n}(W), \mathbb{Z}\right)$. It is proved in $[\mathrm{M}]$ that the map $S$ is given by the intersection form, namely

$$
S(x)(y):=x \cdot y, \quad x, y \in H_{n}(W) .
$$

One also defines the Seifert form of the singularity

$$
A: H_{n}(W) \otimes H_{n}(W) \rightarrow \mathbb{Z}
$$

by $A(x, y):=\operatorname{lk}_{S_{\varepsilon}}\left(x^{+}, y\right)$, where $W$ is now viewed inside $S_{\varepsilon}$ via the Milnor open book given by $f /|f|, x^{+}$denotes a small push-off of $x$ in the positive direction given by the open book decomposition, and $\mathrm{lk}_{S_{\varepsilon}}$ denotes the linking number inside $S_{\varepsilon}$. We then have $S=A+(-1)^{n} A^{t}$ (see [D] and the references therein).

As an immediate consequence of Proposition 2.6, we obtain the following result.

Proposition 6.2. (a) Let $n \geq 3$ and $(\Sigma, \xi)$ be the link of an isolated hypersurface singularity. If the intersection form on the middle-dimensional homology of the Milnor fiber is nonzero, then $(\Sigma, \xi)$ does not embed in a subcritical Stein manifold.

(b) Brieskorn manifolds of dimension $2 n-1, n \geq 3$, with Milnor number at least 2 do not admit contact embeddings in subcritical Stein manifolds.

Proof. (a) The long exact sequence (6.1) shows that surjectivity of the map $H_{n}(\Sigma) \rightarrow$ $H_{n}(W)$ is equivalent to the vanishing of the intersection form. Since $n \geq 3$ we have $H_{2}(W, \Sigma)=0$, and the conclusion follows from Theorem 2.6.

(b) The Brieskorn manifold $\Sigma\left(a_{0}, a_{1}, \ldots, a_{n}\right), a_{0}, \ldots, a_{n} \geq 2$, is, by definition, the link of the singularity $z_{0}^{a_{0}}+\cdots+z_{n}^{a_{n}}=0$. The Milnor number of $\Sigma\left(a_{0}, a_{1}, \ldots, a_{n}\right)$ is $\mu=\left(a_{0}-1\right) \ldots\left(a_{n}-1\right)$. Following [Sa], its Seifert form is the tensor-product of blocks of dimension $a_{i}-1, i=0, \ldots, n$, and the blocks have the form [D]

$$
\left(\begin{array}{ccccc}
1 & 1 & 0 & \ldots & 0 \\
0 & 1 & 1 & \ddots & 0 \\
0 & \ddots & 1 & \ddots & 0 \\
\vdots & & & \ddots & 1 \\
0 & & \ldots & 0 & 1
\end{array}\right) .
$$

Thus $A$ is neither symmetric, nor anti-symmetric, and we infer that $S \neq 0$. The conclusion then follows from (a). 
Remark 6.3. The condition $\mu \geq 2$ is violated if and only if all the exponents $a_{i}$ are equal to 2 . In this case $\Sigma=S T^{*} S^{n}$. For $n$ even the matrix of $A$ is symmetric, hence $S=A+A^{t} \neq 0$, so there is no contact embedding of $\Sigma$ in a subcritical Stein manifold. But an argument of Lisca in [C-F] shows that there is not even a smooth embedding. If $n$ is odd we cannot conclude.

Corollary 6.4. Let $n \geq 3$ and $(\Sigma, \xi)$ be a Brieskorn manifold which is diffeomorphic to the sphere $S^{2 n-1}$. The standard contact structure $\xi$ inherited from the Milnor fiber is exotic, i.e. $\xi$ is not diffeomorphic to the standard contact structure on $S^{2 n-1}$.

Proof. This follows immediately from Proposition 6.2, since $\Sigma$ does not admit a contact embedding in $\mathbb{R}^{2 n}$. There is no need to consider the case $a_{i}=2$ for all $i$ since $S T^{*} S^{n}$ is never diffeomorphic to $S^{2 n-1}$.

Remark 6.5. Ustilovsky has actually exhibited in [U] infinitely many pairwise nonisomorphic contact structures on spheres of dimension $4 m+1$. In [vK], the reader will find an algorithm to compute the linearized contact homology of most Brieskorn manifolds in dimension greater than 5 .

We now consider the manifolds of Mark McLean in [McL]. These are Stein symplectic manifolds $\left(M_{k}^{2 n}, \omega_{k}\right)$ diffeomorphic to $\mathbb{R}^{2 n}(n \geq 4)$, such that $\left(\partial M_{k}^{2 n}, \xi_{k}^{2 n}\right)$ is a contact manifold diffeomorphic to $S^{2 n-1}$. However, McLean shows that $S H_{n}\left(M_{k}^{n}\right)$ contains $N^{k}$ idempotent elements for some $N \geq 2$, therefore the manifolds $M_{k}^{2 n}$ are pairwise non symplectomorphic.

We now prove

Proposition 6.6. The contact manifolds $\left(\partial M_{k}^{2 n}, \xi_{k}^{2 n}\right)$ are never contactomorphic to the standard sphere.

Proof. Let us denote for simplicity $W=M_{k}^{2 n}$ and $(\Sigma, \xi)=\left(\partial M_{k}^{2 n}, \xi_{k}^{2 n}\right)$. The exact sequence in symplectic homology reads

$$
\longrightarrow H_{2 n}(W, \Sigma) \longrightarrow S H_{n}(W) \longrightarrow S H_{n}^{+}(W) \longrightarrow 0 \text {. }
$$

Assume $(\Sigma, \xi)$ is the standard sphere. Then $S H_{n}^{+}(W)$ only depends on $(\Sigma, \xi)$ so is the same as $S H_{n}^{+}\left(D^{2 n}\right)=0$. As a result we should have $\operatorname{rank}\left(S H_{n}(W)\right) \leq 1$. But for $k \geq 2$, there are at least 3 idempotents, hence the rank is at least 2 and we get a contradiction.

If we knew that there is a contact form on $\left(\partial M_{k}^{2 n}, \xi_{k}^{2 n}\right)$ with no closed characteristic of index less than $3-n$, then we would get, by the above argument, that $\left(\partial M_{k}^{2 n}, \xi_{k}^{2 n}\right)$ has no embedding in an SAWC manifold. 


\section{Summary}

A conceptual framework for the study of symplectic fillings is provided by the following definition of [Et-Ho].

Definition 7.1 ([Et-Ho]). Let $\left(\Sigma_{1}, \xi_{1}\right)$ and $\left(\Sigma_{2}, \xi_{2}\right)$ be two closed contact manifolds. We say that $\left(\Sigma_{1}, \xi_{1}\right)$ is dominated by $\left(\Sigma_{2}, \xi_{2}\right)$ if there exists a symplectically aspherical manifold $(W, \omega)$ such that $(W, \omega)$ has $\left(\Sigma_{1}, \xi_{1}\right)$ as a concave boundary, $\left(\Sigma_{2}, \xi_{2}\right)$ as a convex boundary and no other boundary component. We shall write

$$
\left(\Sigma_{1}, \xi_{1}\right) \prec\left(\Sigma_{2}, \xi_{2}\right)
$$

We shall say that $\left(\Sigma_{1}, \xi_{1}\right)$ is equivalent to $\left(\Sigma_{2}, \xi_{2}\right)$ if we both have $\left(\Sigma_{1}, \xi_{1}\right) \prec\left(\Sigma_{2}, \xi_{2}\right)$ and $\left(\Sigma_{2}, \xi_{2}\right) \prec\left(\Sigma_{1}, \xi_{1}\right)$, and this is denoted by

$$
\left(\Sigma_{1}, \xi_{1}\right) \simeq\left(\Sigma_{2}, \xi_{2}\right)
$$

Remark 7.2. In the terminology of Symplectic Field Theory, we see that $\left(\Sigma_{1}, \xi_{1}\right)$ is dominated by $\left(\Sigma_{2}, \xi_{2}\right)$ if and only if there exists a symplectically aspherical cobordism between $\left(\Sigma_{1}, \xi_{1}\right)$ and $\left(\Sigma_{2}, \xi_{2}\right)$.

Clearly, we have

$$
\left(\Sigma_{1}, \xi_{1}\right) \simeq\left(\Sigma_{1}, \xi_{1}\right)
$$

We would like to know if there are nonequivalent pairs of contact manifolds. Clearly, a contact manifold admits a filling if and only if it dominates the standard sphere. Which manifolds are dominated by the standard sphere? Our results give examples of fillable manifolds which are not dominated by the standard sphere or, more generally, by the boundary of a subcritical Stein manifold. On the other hand, in dimension 4, any overtwisted contact manifold is dominated by any other contact manifold (see [Et-Ho]). In particular, all overtwisted contact structures are equivalent!

The point of view of Definition 7.1 is also related to the work of [Ch] on the non-symmetry of Legendrian concordances.

We here try to summarize our results, but warn the reader that in the table below, the assumptions of the theorems are usually incomplete and the statements often not precise. One should refer to the relevant section of the paper for full details.

Acknowledgements. We are grateful to Vincent Blanlœil for his help with the formulation and proof of Proposition 6.2. We thank the anonymous referee for many useful questions and for contributing to improve our manuscript. 


\begin{tabular}{|c|c|c|c|c|c|c|c|c|}
\hline \multicolumn{2}{|r|}{ 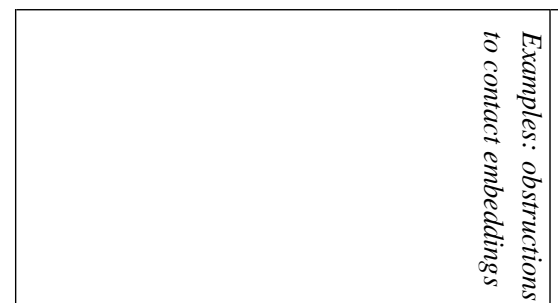 } & 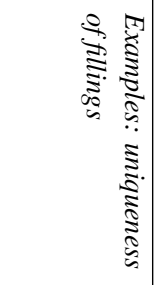 & 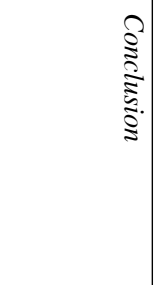 & 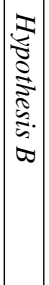 & 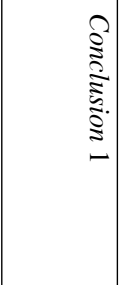 & & 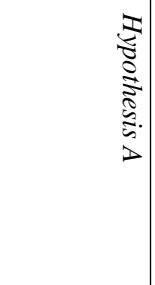 & \\
\hline \multicolumn{2}{|l|}{ 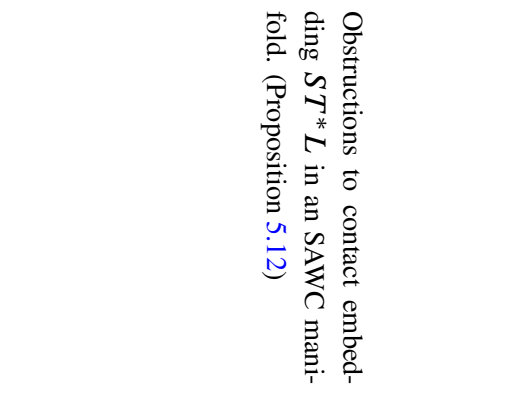 } & & 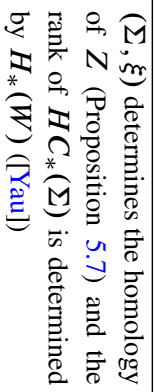 & 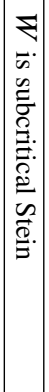 & & & 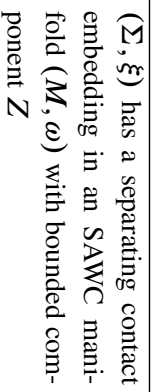 & 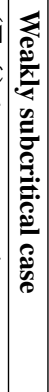 \\
\hline \multirow[t]{2}{*}{ 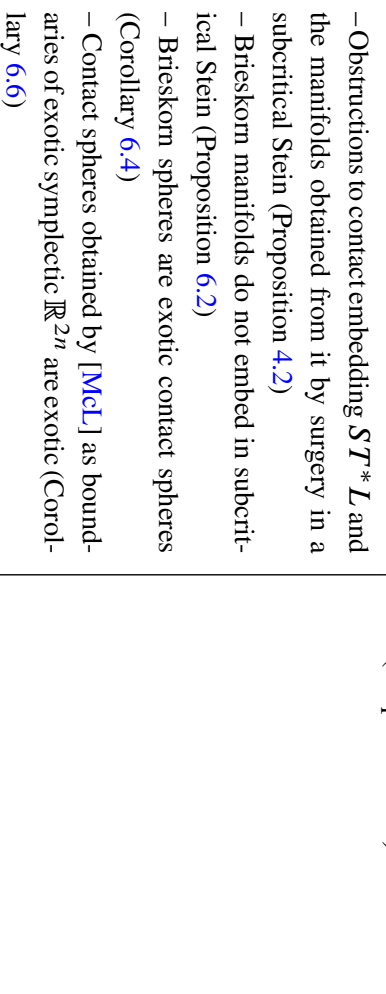 } & 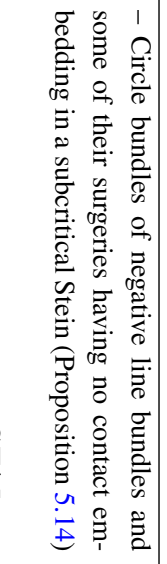 & 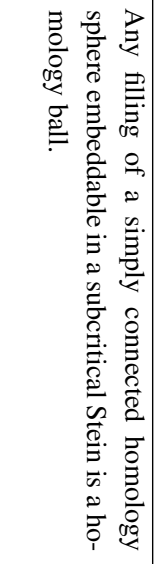 & 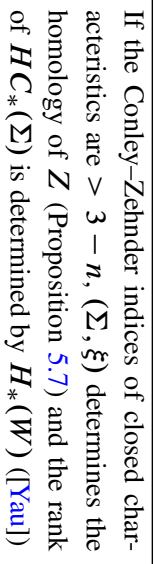 & 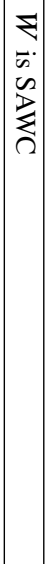 & 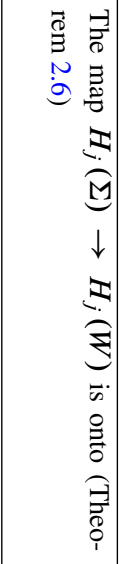 & 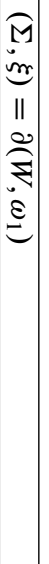 & 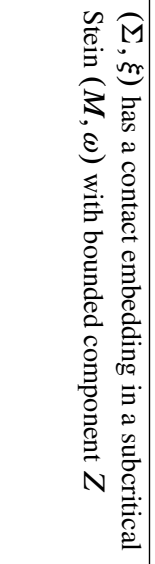 & 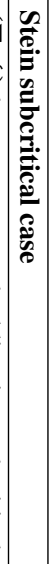 \\
\hline & 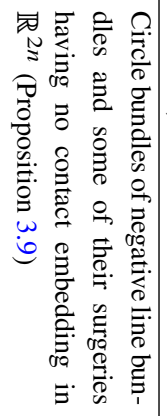 & 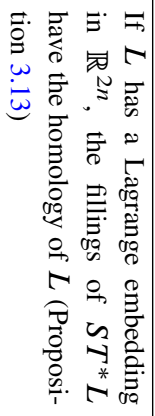 & 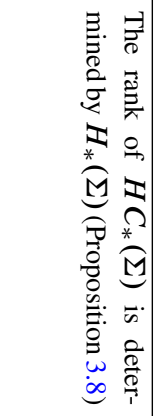 & 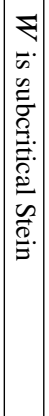 & 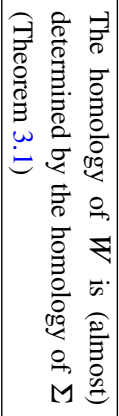 & 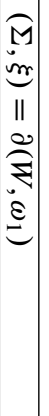 & 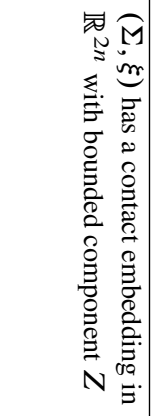 & 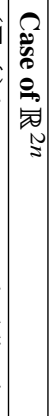 \\
\hline
\end{tabular}




\section{References}

[A-McL] P. Albers and M. McLean, Non-displaceable contact embeddings and infinitely many leaf-wise intersections. J. Symplectic Geom. 9 (2011), no. 3, 271-284. Zbl 05956508 MR 2817777

[Bi] P. Biran, Symplectic topology and algebraic families. In Proceedings of the European Congress of Mathematics (Stockholm 2004), 827-836. Zbl 1078.53087 MR 2185784

[B-O-1] F. Bourgeois and A. Oancea, An exact sequence for contact- and symplectic homology. Invent. Math. 175 (2009), 611-680. Zbl 1167.53071 MR 2471597

[B-O-2] F. Bourgeois and A. Oancea, The Gysin exact sequence for $S^{1}$-equivariant symplectic homology. arXiv:0909.4526 [math.SG]

[Ch] B. Chantraine, Lagrangian concordance of Legendrian knots. Algebr. Geom. Topol. 10 (2010), no. 1, 63-85. Zbl 1203.57010 MR 2580429

[C1] K. Cieliebak, Subcritical Stein manifolds are split. arXiv:math.DG/0204351 [math.DG]

[C2] K. Cieliebak, Handle attaching in symplectic homology and the chord conjecture. J. Eur. Math. Soc. (JEMS) 4 (2002), 115-142 . Zbl 1012.53066 MR 1911873

[C-F] K. Cieliebak and U. Frauenfelder, A Floer homology for exact contact embeddings. Pacific J. Math. 239 (2009), 251-316. Zbl 05541980 MR 2461235

[C-F-O] K. Cieliebak, U. Frauenfelder, and A. Oancea, Rabinowitz Floer homology and symplectic homology. Ann. Sci. Éc. Norm. Supér. (4) 43 (2010), no. 6, 957-1015. Zbl 1213.53105 MR 2778453

[D] A. Durfee, Fibered knots and algebraic singularities. Topology 13 (1974), 47-59. Zbl 0275.57007 MR 0336750

[E] Y. Eliashberg, Topological characterization of Stein manifolds of dimension $>2$. Internat. J. Math. 1 (1990), 29-46. Zbl 0699.58002 MR 1044658

[Et-Ho] J. Etnyre and K. Honda, On Symplectic Cobordisms. Math. Ann. 323 (2002), 31-39. Zbl 1022.53059 MR 1906906

[G-Ha] M. Greenberg and J. Harper, Algebraic topology: a first course. BenjaminCummings Pub. Comp., London 1981. Zbl 0498.55001 MR 0643101

[Gr] M. Gromov, Pseudo-holomorphic curves in symplectic manifolds. Invent. Math. 82 (1985), 307-347. Zbl 0592.53025 MR 0809718

[La] F. Laudenbach, Trois constructions en topologie symplectique. Ann. Fac. Sci. Toulouse Math. 6 (1997), 697-709. Zbl 0947.57033 MR 1624314

[Li-M] P. Lisca and G. Matić, Tight contact structures and Seiberg-Witten invariants. Invent. Math. 129 (1997), 509-525. Zbl 0882.57008 MR 1465333

[L-McD-S] L. Lazzarini, D. McDuff, and D. Salamon, Singularities and intersections. Appendix E of $J$-holomorphic curves and symplectic topology by D. McDuff and D. Salamon, Amer. Math. Soc. Colloq. Publ. 52, Amer. Math. Soc., Providence, RI, 2004, 601-642. Zbl 1064.53051 MR 2045629

[McD] D. McDuff, Symplectic manifolds with contact type boundary. Invent. Math 103 (1991), 651-671. Zbl 0719.53015 MR 1091622 
Vol. 87 (2012) On the topology of fillings of contact manifolds and applications

[McL] M. McLean, Lefschetz fibrations and symplectic homology. Geom. Topol. 13 (2009), 1877-1944. Zbl 1170.53070 MR 2497314

[M] J. W. Milnor, Singular points of complex hypersurfaces. Ann. of Math. Stud. 61, Princeton University Press, Princeton, N.J., 1968. Zbl 0184.48405 MR 0239612

[O] A. Oancea, Fibered symplectic cohomology and the Leray-Serre spectral sequence. J. Symplectic Geom. 6 (2008), no. 3 267-351. Zbl 1157.57016 MR 2448827

[Po] P. Popescu-Pampu, On the cohomology rings of holomorphically fillable manifolds. In Singularities II. Geometric and topological aspects, ed. by J. P. Brasselet et al., Contemp. Math. 475, Amer. Math. Soc., Providence, RI, 2008, 169-188. Zbl 1161.32015 MR 2454366

[Sa] K. Sakamoto, The Seifert matrices of Milnor fiberings defined by holomorphic functions. J. Math. Soc. Japan 26 (1974), 714-721. Zbl 0286.32010 MR 0362329

[Se] P. Seidel, Symplectic homology as Hochschild homology. In Algebraic geometrySeattle 2005, ed. by D. Abramovich et al. Proc. Sympos. Pure Math. 80, Part 1, Amer. Math. Soc., Providence, RI, 2009, 415-434. Zbl 1179.53085 MR 2483942

[Sm] S. Smale, On the structure of manifolds. Amer. J. Math. 84 (1962), 387-399. Zbl 0109.41103 MR 0153022

[U] I. Ustilovsky, Infinitely many contact structures on $S^{4 m+1}$. Internat. Math. Res. Notices 14 (1999), 781-791. Zbl 1034.53080 MR 1704176

[vK] O. van Koert, Contact homology of Brieskorn manifolds. Forum Math. 20 (2008), 317-339. Zbl 1155.57025 MR 2395966

[V] C. Viterbo, Functors and computations in Floer homology with applications. Part I. Geom. Funct. Anal. 9 (1999), 985-1033. Zbl 0954.57015 MR 1726235

[W] A. Weinstein, Contact surgery and symplectic handlebodies. Hokkaido Math. J. 20 (1991), 241-251. Zbl 0737.57012 MR 1114405

[Yau] M.-L. Yau, Cylindrical contact homology of subcritical Stein fillable contact manifolds. Geom. Topol. 8 (2004), 1243-1280. Zbl 1055.57036 MR 2087083

Received May 8, 2009

Alexandru Oancea, IRMA, UMR 7501, Université de Strasbourg, 7, rue René Descartes, 67084 Strasbourg, France

E-mail: oancea@math.u-strasbg.fr

Claude Viterbo, Centre de Mathématiques Laurent Schwartz, UMR 7640 du CNRS, École Polytechnique, 91128 Palaiseau, France; current address: DMA-Ecole Normale Supérieure, 45, rue d'Ulm, 75230 Paris Cedex 05, France

E-mail: viterbo@dma.ens.fr 